\title{
Interference contributions to gluon initiated heavy Higgs production in the two-Higgs-doublet model
}

\author{
Nicolas Greiner ${ }^{1,2, a}$, Stefan Liebler ${ }^{1, b}$, Georg Weiglein ${ }^{1, \mathrm{c}}$ \\ ${ }^{1}$ DESY, Notkestraße 85, 22607 Hamburg, Germany \\ 2 Physik-Institut, Universität Zürich, Winterthurerstrasse 190, 8057 Zürich, Switzerland
}

Received: 5 January 2016 / Accepted: 21 February 2016 / Published online: 3 March 2016

(c) The Author(s) 2016. This article is published with open access at Springerlink.com

\begin{abstract}
We discuss the production of a heavy neutral Higgs boson of a $\mathcal{C P}$-conserving two-Higgs-doublet model in gluon fusion and its decay into a four-fermion final state, $g g(\rightarrow V V) \rightarrow e^{+} e^{-} \mu^{+} \mu^{-} / e^{+} e^{-} \nu_{l} \bar{\nu}_{l}$. We investigate the interference contributions to invariant mass distributions of the four-fermion final state and other relevant kinematical observables. The relative importance of the different contributions is quantified for the process in the on-shell approximation, $g g \rightarrow Z Z$. We show that interferences of the heavy Higgs with the light Higgs boson and background contributions are essential for a correct description of the differential cross section. Even though they contribute below $\mathcal{O}(10 \%)$ to those heavy Higgs signal cross sections, to which the experiments at the Large Hadron Collider were sensitive in its first run, we find that they are sizable in certain regions of the parameter space that are relevant for future heavy Higgs boson searches. In fact, the interference contributions can significantly enhance the experimental sensitivity to the heavy Higgs boson.
\end{abstract}

\section{Introduction}

The two multi-purpose experiments ATLAS and CMS at the CERN Large Hadron Collider (LHC) discovered in 2012 a scalar resonance at $125 \mathrm{GeV}$ [1,2], which is compatible with a Standard Model (SM) Higgs boson. Even though its couplings are - to the precision obtained so far - in agreement with the SM expectations, it can well be embedded in an extended Higgs sector like a two-Higgs-doublet model (2HDM).

\footnotetext{
a e-mail: greiner@physik.uzh.ch

be-mail: stefan.liebler@desy.de

c e-mail: georg.weiglein@desy.de
}

The main production mechanism for a SM-like Higgs boson $h$ is gluon fusion [3], for which a large amount of higher-order corrections in quantum chromodynamics (QCD) [4-11] are known. Recently the combined scale and $\mathrm{PDF}+\alpha_{s}$ uncertainties, see e.g. Refs. [12-14], were reduced to below $\mathcal{O}(10) \%$. Of particular importance for the discovery of the new particle and the subsequent investigations of its mass and couplings were the decays into heavy gauge bosons $h \rightarrow V V$ with $V \in\{W, Z\}$, which appear to be of relevance also for off-shell Higgs bosons [15] (see also Ref. [16] for an investigation of off-shell contributions at a linear collider and the LHC). Given the small theory uncertainties and because of their importance for the unitarization of the process, interference effects between the off-shell Higgs boson and the continuum background in $g g \rightarrow h^{*} \rightarrow V V$ were studied for leptonic decays in Refs. [17-30]. The semileptonic process, where interferences with tree-level background diagrams occur, was recently discussed for the first time [31]. Whereas for the processes $g g \rightarrow h h, g g \rightarrow h Z$ and $g g \rightarrow \gamma \gamma$ nextto-leading order (NLO) QCD contributions have been known for quite some time [32-34], the calculation of background contributions to $g g \rightarrow V V$ for off-shell gauge bosons in the limit of massless quarks at NLO QCD became available just very recently [35-38]. For the mentioned signal-background interference approximate higher order contributions using a soft-collinear approximation for a heavy Higgs boson [29] and applying soft-gluon resummation for an off-shell light Higgs boson [30] were previously available.

We discuss $g g \rightarrow(V V) \rightarrow e^{+} e^{-} \mu^{+} \mu^{-} / e^{+} e^{-} \nu_{l} \bar{\nu}_{l}$ at LO QCD in the context of a $\mathcal{C P}$-conserving $2 \mathrm{HDM}$, see Refs. [39-44] for 2HDM reviews. We furthermore assume the absence of tree-level flavor-changing neutral currents. The Higgs sector of the $\mathcal{C} \mathcal{P}$-conserving $2 \mathrm{HDM}$ consists of three Higgs bosons $\phi_{i}, i \in\{1,2,3\}$, namely two $\mathcal{C P}$-even Higgs bosons $h, H$ with masses $m_{h}<m_{H}$ and one $\mathcal{C P}$-odd Higgs boson $A$. According to the structure of the Yukawa couplings four types of 2HDMs are distinguished, where for 
our purposes only two types are of relevance, namely those with different couplings of the two Higgs doublets to up- and down-type quarks. The process $g g \rightarrow V V$ was only very recently discussed in the context of a 2 HDM [45], following a discussion of $g g \rightarrow t \bar{t}$ [46]. In contrast, for the similar processes $g g \rightarrow \phi_{i} \phi_{j}$ [47] and $g g \rightarrow Z \phi_{i}$ [48] in the 2HDM even higher order effects were partially included. The process $g g \rightarrow V V$ was recently extensively discussed in the context of the SM with an additional real singlet $[49,50]$, and also the vector-boson fusion process was considered in Ref. [51]. The extension of the SM by a real singlet can be characterized by a single angle which multiplies all Higgs couplings to quarks, leptons, and gauge bosons with a universal factor. Accordingly the phenomenology of the $2 \mathrm{HDM}$ is more rich, since in the 2HDM in particular the couplings of the two $\mathcal{C P}$-even Higgs bosons to quarks and to gauge bosons are modified differently.

The interference contributions to the process $g g \rightarrow$ $(V V) \rightarrow e^{+} e^{-} \mu^{+} \mu^{-} / e^{+} e^{-} \nu_{l} \bar{\nu}_{l}$, within the 2HDM, which we study in the present paper, are of interest for several reasons. The interference contributions of the heavy Higgs with the light Higgs and the background are crucial for the unitarization of the process. The related effects are particularly important for high invariant masses of the gauge bosons, i.e. at high energies of the hard scattering process. In general, the interference effects need to be well understood in order to obtain a sufficiently accurate prediction for the process. Furthermore, interference effects are of interest since they can potentially enhance the sensitivity to the signal of a heavy Higgs boson. All those aspects are addressed in our analysis below.

We make use of GoSam $[52,53]$ to discuss the processes $g g \rightarrow e^{+} e^{-} \mu^{+} \mu^{-}$and $e^{+} e^{-} \nu_{l} \bar{\nu}_{l}$ (including all three neutrino flavors). For a study of the relevance of interference contributions we also consider the case where the first process is approximated by the on-shell production of two $Z$ boson, $g g \rightarrow Z Z$ (and the subsequent decays of the $Z$ bosons). We added its amplitudes for this process to a modified version [54] of vh@nnlo [55], which has been linked to $2 \mathrm{HDMC}$ [56].

The LHC experiments recently presented results for a heavy Higgs search with subsequent decay into heavy gauge bosons in Refs. [57,58]. The ATLAS experiment also provided an interpretation in terms of the $2 \mathrm{HDM}$, neglecting possible interferences between the heavy Higgs signal and the background as well as with the contribution of the light Higgs boson. The CMS experiment took into account a rescaled interference from the SM case of a heavy Higgs boson. Neglecting the interference contributions involving the heavy Higgs boson and employing the narrow-width approximation (NWA) for the heavy Higgs boson, $g g \rightarrow H \rightarrow V V$, has of course the advantage that all known QCD and electroweak corrections for gluon fusion and the decay into heavy quarks as implemented in codes like SusHi [59] or Prophecy $4 F[60,61]$ can be taken into account. In our analysis of the interference contributions to the process $g g \rightarrow(V V) \rightarrow e^{+} e^{-} \mu^{+} \mu^{-} / e^{+} e^{-} \nu_{l} \bar{\nu}_{l}$ we find that neglecting the interference contributions of the heavy Higgs boson with the background and the light Higgs boson in the ATLAS analysis has indeed been justified in view of the experimental sensitivity that has been reached in the first run of the LHC. On the other hand, we find that these interference contributions will be of relevance for high integrated luminosities at the LHC.

With respect to the interference contributions involving the heavy Higgs boson it is obviously important to ensure that the cross section into heavy gauge bosons is correctly unitarized, in particular at high invariant masses of the gauge boson system. In case interferences involving the heavy Higgs boson are neglected the light Higgs boson and its interference with the background has to have SMlike Higgs boson couplings. For the pure signal strength of a heavy Higgs boson Ref. [45] suggests to use a multiplicative factor covering the interference effects in the context of a 2 HDM. We find it preferable to take into account all interferences of the light Higgs boson, the heavy Higgs boson and the background consistently in the setup of a 2HDM in order to describe the cross section at high invariant masses accurately, as done in the present paper. We find that in the vicinity of the heavy Higgs boson mass peak the role of interferences is essential since they simultaneously alter the form and the position of the heavy Higgs boson mass peak. We furthermore show that in certain regions of the parameter space the interferences of the heavy Higgs boson with the light Higgs boson and the background significantly enhance the heavy Higgs boson signal and thus increase the experimental sensitivity in heavy Higgs boson searches.

The paper is organized as follows: We explain the theoretical background in Sect. 2 starting with a short introduction to the two-Higgs-doublet model and providing the details of our cross section calculations for the processes under consideration. In Sect. 3 we discuss the 2 HDM scenarios that we consider in our study, and briefly describe the employed selection cuts. Lastly, we present our numerical results in Sect. 4 and conclude in Sect. 5.

\section{Theoretical background}

In this section we first discuss the basics of the two-Higgsdoublet model (2HDM) and afterwards describe our implementation of the processes $g g \rightarrow Z Z$ and $g g \rightarrow e^{+} e^{-}$ $\mu^{+} \mu^{-} / e^{+} e^{-} \nu_{l} \bar{\nu}_{l}$ with the help of FeynArts, FormCalc and GoSam. 
Table 1 Relative couplings $g_{f}^{\phi}$ (with respect to the SM coupling) for the two $2 \mathrm{HDM}$ types

\begin{tabular}{lllll}
\hline Model & $g_{u}^{h}$ & $g_{d}^{h}$ & $g_{u}^{H}$ & $g_{d}^{H}$ \\
\hline Type I & $\cos \alpha / \sin \beta$ & $\cos \alpha / \sin \beta$ & $\sin \alpha / \sin \beta$ & $\sin \alpha / \sin \beta$ \\
Type II & $\cos \alpha / \sin \beta$ & $-\sin \alpha / \cos \beta$ & $\sin \alpha / \sin \beta$ & $\cos \alpha / \cos \beta$ \\
\hline
\end{tabular}

\subsection{Two-Higgs-doublet model}

The 2HDM contains two Higgs doublets, which we name $H_{1}$ and $H_{2}$. It can conveniently be classified in four types if one demands the absence of tree-level flavor-changing neutral currents and furthermore assumes $\mathcal{C P}$ conservation. By convention, the up-type quarks couple to $\mathrm{H}_{2}$, such that the couplings to the down-type quarks and to the leptons can either be through $H_{1}$ or $H_{2}$, which corresponds to the four different types. For details we refer to Refs. [39-44]. Since our studies are not sensitive to the coupling of the Higgs bosons to leptons, it is sufficient to restrict our discussion to the two types I and II. The two Higgs doublets form one $\mathcal{C P}$ odd field $A$ and two $\mathcal{C P}$-even Higgs fields $h$ and $H$ due to $\mathcal{C P}$ conservation, as well as two charged Higgs bosons $H^{ \pm}$. The 2HDM can be described in different basis representations. We make use of the "physical basis", in which the masses of all physical Higgs bosons, the ratio of the vacuum expectation values $t_{\beta}:=\tan \beta=v_{2} / v_{1}$ and the Higgs mixing angle in the $\mathcal{C P}$-even sector $\alpha$, or alternatively $s_{\beta-\alpha}:=\sin (\beta-\alpha)$, are taken as input parameters. Together with the mass term $m_{12}^{2}$ of both Higgs bosons $H_{1}^{\dagger} H_{2}$ all parameters of the Higgs sector of a $2 \mathrm{HDM}$ are fixed. We choose $\beta-\alpha$ in between $-\pi / 2 \leq \beta-\alpha \leq \pi / 2$, such that $-1 \leq s_{\beta-\alpha} \leq 1$ and $0 \leq c_{\beta-\alpha} \leq 1$. Our scenarios are thus specified by the two angles $\alpha$ and $\beta$, which completely determine the relative couplings (with respect to the couplings of a SM Higgs boson) of the light and the heavy Higgs boson to quarks and the heavy gauge boson. They are provided in Eq. (1) and Table 1 (together with Eq. (2) for a decomposition in terms of $\beta-\alpha$ and $\beta$ ). Moreover, our analysis is sensitive to $m_{h}$ and $m_{H}$, whereas it is rather insensitive to the mass of the pseudoscalar $m_{A}$ and the heavy charged Higgs boson mass $m_{H^{ \pm}}$, as long as they are heavy enough not to open decay modes of the heavy Higgs $H$ into them and as long as the decay mode $H \rightarrow h h$ is sub-dominant. If the latter condition is fulfilled, also the dependence on the mass term $m_{12}^{2}$ can be neglected.

The Higgs boson couplings to the gauge bosons $V \in$ $\{W, Z\}$ relative to the $\mathrm{SM}$ are given by

$g_{V}^{h}=\sin (\beta-\alpha)=: s_{\beta-\alpha}, \quad g_{V}^{H}=\cos (\beta-\alpha)=: c_{\beta-\alpha}$.

The pseudoscalar has no lowest-order couplings to a pair of gauge bosons. It can in principle contribute to the considered processes with four fermions in the final state. Because of the suppression of the Yukawa couplings to leptons, how- ever, these contributions are very small, and thus diagrams involving the pseudoscalar are not of relevance for our discussion. The relative couplings of the heavy Higgs boson to bottom quarks and top quarks, which are of particular relevance for our discussion, are given by

$$
g_{t}^{H}=\frac{\sin \alpha}{\sin \beta}=-s_{\beta-\alpha} \frac{1}{t_{\beta}}+c_{\beta-\alpha},
$$

Type I: $g_{b}^{H}=\frac{\sin \alpha}{\sin \beta}=-s_{\beta-\alpha} \frac{1}{t_{\beta}}+c_{\beta-\alpha}$,

Type II: $g_{b}^{H}=\frac{\cos \alpha}{\cos \beta}=s_{\beta-\alpha} t_{\beta}+c_{\beta-\alpha}$.

In the decoupling limit, $\left|s_{\beta-\alpha}\right| \rightarrow 1$, the light Higgs boson $h$ couples to the gauge bosons with the same strength as the SM Higgs boson. In contrast, the more SM-like the coupling of the light Higgs boson to gauge bosons is, the more suppressed is the coupling of the heavy Higgs boson $g_{V}^{H}$ to gauge bosons, as a consequence of the well-known sum rule $\left(g_{V}^{h}\right)^{2}+\left(g_{V}^{H}\right)^{2}=1$. Accordingly, for a 2HDM with a SMlike Higgs boson $h$ at $125 \mathrm{GeV}$ the signal for the production of the heavier Higgs $H$ in $g g \rightarrow H \rightarrow V V$ will necessarily be rather weak.

For a suppressed signal of this kind interference effects can be important, both with the off-shell light Higgs boson contribution as well as with the background diagrams. Our analysis will quantify these effects for different final states as a function of $s_{\beta-\alpha}, t_{\beta}$ as well as the employed Higgs mass $m_{H}$. In the context of interference effects obviously also the width, $\Gamma_{H}$, is an important quantity. In our analysis $\Gamma_{H}$ is not treated as a free parameter, but it is calculated from the other 2HDM parameters with the help of 2HDMC.

\subsection{Details of the calculation}

We briefly describe here the calculations that we have carried out for the process with two on-shell $Z$ bosons, $g g \rightarrow Z Z$, and for the full process with four fermions in the final state, $g g \rightarrow e^{+} e^{-} \mu^{+} \mu^{-}, g g \rightarrow e^{+} e^{-} v_{\mu} \bar{v}_{\mu}$ and $g g \rightarrow e^{+} e^{-} v_{e} \bar{v}_{e}$. Our implementation of $g g \rightarrow Z Z$ within vh@nnlo was generated with the help of FeynArts [62] and FormCalc [63], see the diagrams in Fig. 1. The implementation links to LoopTools [63] for the calculation of the employed one-loop Feynman integrals and to 2HDMC [56] for the calculation of the Higgs boson widths $\Gamma_{h}$ and $\Gamma_{H}$. In comparison to our treatment of the full process, see below, we employ additional approximations for the process 


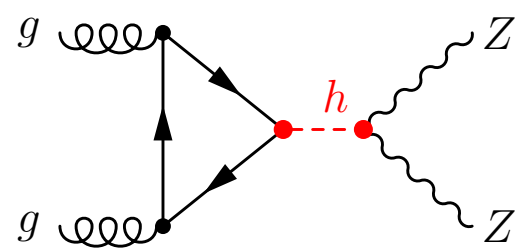

(a)

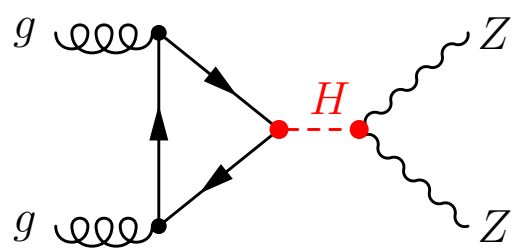

(b)

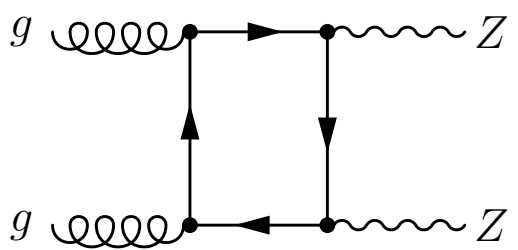

(c)

Fig. 1 Feynman diagrams for $g g \rightarrow Z Z$

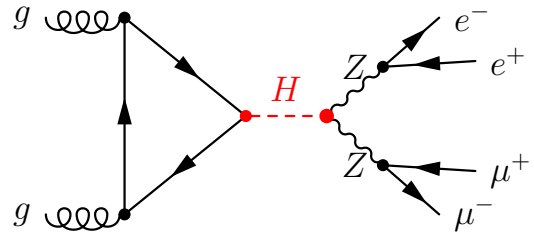

(a)

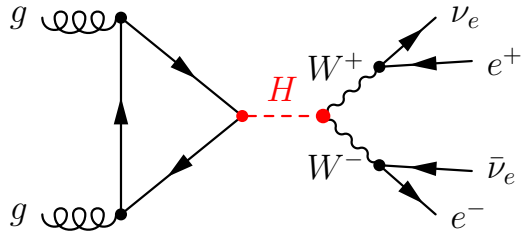

(b)

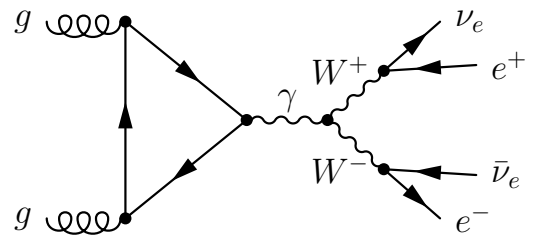

(c)

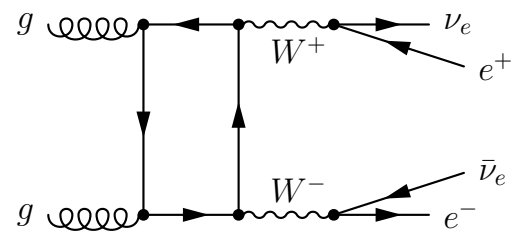

(d)

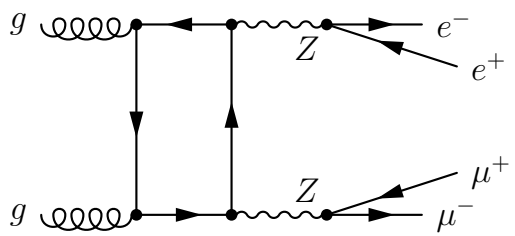

(e)

Fig. 2 Sample Feynman diagrams for double resonant $W, Z$ contributions, i.e. where the final state leptons directly come from the decay of massive gauge bosons

$g g \rightarrow Z Z$, for which we investigate the different interference contributions. In particular, we do not take into account the top-quark contribution to the box diagrams in $g g \rightarrow Z Z$, see Fig. 1c, even though they are of relevance for large invariant masses of the gauge bosons above $2 m_{t}$. However, the top-quark contribution to those diagrams does not add new features to our qualitative discussion of interference effects in Sect. 4.2. We treat the remaining five quarks massless in contrast to our GoSam implementation, which includes all six quarks with finite top- and bottom-quark masses, see below. The triangle diagrams with an intermediate light or heavy Higgs boson, see Fig. 1a, b, take into account the massive top-quark and bottom-quark contributions.

The amplitudes for the processes $g g \rightarrow e^{+} e^{-} \mu^{+} \mu^{-}$, $g g \rightarrow e^{+} e^{-} v_{\mu} \bar{v}_{\mu}$ and $g g \rightarrow e^{+} e^{-} \nu_{e} \bar{v}_{e}$ have been generated with GoSam [52,53]. GoSam is a publicly available tool for the automated generation of one-loop amplitudes within and beyond the Standard Model. It is based on a Feynman diagrammatic approach, where the Feynman diagrams are first generated with QGraf [64] and Form [65, 66], and Spinney [67], Haggies [68] and Form are used to write an optimized Fortran output. For the reduction of the tensor integrals there are several options available. We used Ninja [69-71], an automated package for integrand reduction via Laurent expansion. Alternatively one can use other reduction techniques such as integrand reduction in the OPP method [72-74] as implemented in Samurai [75] or methods of tensor integral reduction as implemented in Golem95 [76-79]. The resulting scalar integrals are evaluated using OneLOop [80].

In this case the implementation of a 2HDM model in GoSam requires only the implementation of a second Higgs boson, while leaving the relative couplings $g_{f}^{h, H}$ and $g_{V}^{h, H}$ as free parameters, which can be modified according to the specific parameters that are considered. Our discussion including the different decay channels of the intermediate vector bosons considers final states with four leptons, namely

$$
\begin{aligned}
& g g \rightarrow e^{+} e^{-} \mu^{+} \mu^{-}, \quad g g \rightarrow e^{+} e^{-} \nu_{\mu / \tau} \bar{\nu}_{\mu / \tau}, \\
& \quad g g \rightarrow e^{+} e^{-} \nu_{e} \bar{\nu}_{e},
\end{aligned}
$$

where we have to sum over all possible intermediate configurations leading to the given final state. This particularly means that depending on the subprocess, also intermediate $W$ bosons as well as non-resonant contributions and photon exchange have to be taken into account. In Fig. 2 we show a few sample diagrams that contribute to a resonant decay of the massive gauge bosons, either for the actual signal (including a Higgs boson) or to the background (without an intermediate Higgs boson exchange). 


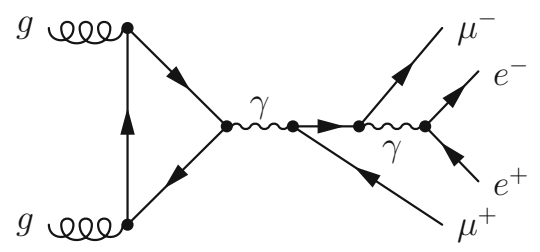

(a)

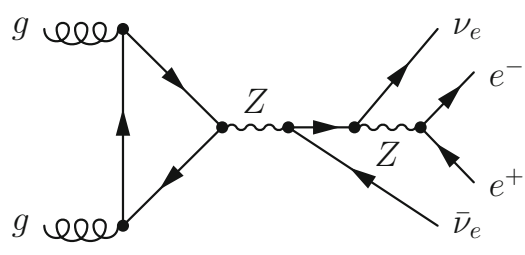

(b)

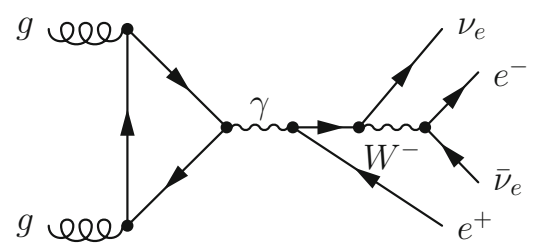

(c)

Fig. 3 Sample Feynman diagrams for single- and non-resonant $W, Z$ contributions for the subprocesses under consideration

In Fig. 3 we show a few sample diagrams for single- or non-resonant $W, Z$ contributions to the same four lepton final states. These types of contributions make it necessary to impose certain cuts on the final state leptons to render the cross section finite, see below.

For the box diagrams, see e.g. Fig. 2 d, e, we take into account all six quark flavors, where the first four are considered to be massless. For diagrams involving a Higgs coupling to quarks only top and bottom quarks are of relevance, since lighter quark contributions are suppressed by their small Yukawa couplings. For the numerical integration over the four particle phase space we have combined the GoSam amplitudes with the integration routines provided by MadEvent [81,82].

It is well known that the calculation of processes including internal Higgs bosons, in particular if one includes higher orders, needs a gauge-invariant formulation of the Higgs boson propagator. Since we are working at LO QCD only, a simplistic Breit-Wigner propagator is sufficient for all our purposes.

We checked our modified vh@nnlo and our GoSam implementations against each other for $g g \rightarrow Z Z$ at the amplitude level and reproduced parts of the results presented in Ref. [50] for the four leptonic final state within the numerical uncertainties.

While for the purpose of brevity of our presentation we do not give explicit analytical results for the final states with four fermions, we refer the reader to Ref. [18] for the helicity amplitudes of $g g \rightarrow Z Z$ (taking into account the correction described in Ref. [45]). The amplitudes given for the SM can individually be translated to the $2 \mathrm{HDM}$ case by multiplying them with effective couplings of Higgs bosons to fermions and gauge bosons. The split in longitudinally and transversely polarized final state $Z$ bosons is instructive, since the relative fraction of longitudinal $Z$ bosons rises with increasing invariant masses of the two $Z$ bosons. This rise also explains the relevance of the top-quark contribution for the background amplitudes involving longitudinal $Z$ bosons, since they are proportional to $m_{t}^{2}$. We focus in the following on our numerical analysis in order to discuss the dependences on all relevant 2HDM parameters. For a discussion of some of the qualitative features in terms of contributions from real and imaginary parts to the interferences we also refer to Ref. [45].
In our numerical analysis below we will first study the full process with four fermions in the final state generated with GoSam in order to discuss the experimental sensitivities in terms of the relevant distributions. In a second step we will focus on the on-shell approximation for two $Z$ bosons, i.e. the simplified process $g g \rightarrow Z Z$, in order to quantify the relevance of interference contributions.

\section{Parameter choice and selection cuts}

We consider five benchmark scenarios to cover different aspects of a heavy Higgs boson in the phenomenology of a 2 HDM. The scenarios yield sufficiently high event rates for the heavy Higgs boson to be potentially observable at the LHC at least at very high integrated luminosities. We also vary certain parameters of the scenarios in order to discuss the relevance of the interference of the heavy Higgs signal with the light Higgs boson and the background. All scenarios include a light Higgs boson with mass $m_{h}=125 \mathrm{GeV}$. We keep the couplings of the light Higgs close to the ones of the SM Higgs by a proper choice of $t_{\beta}$ and $s_{\beta-\alpha}$. The masses (and widths) of quarks and gauge bosons are set to

$$
\begin{aligned}
& m_{t}=172.3 \mathrm{GeV}, \quad m_{b}\left(m_{b}\right)=4.16 \mathrm{GeV}, \\
& m_{z}=91.1876 \mathrm{GeV}, \quad m_{w}=80.398 \mathrm{GeV}, \\
& \Gamma_{z}=2.4952 \mathrm{GeV}, \quad \Gamma_{w}=2.085 \mathrm{GeV} .
\end{aligned}
$$

To keep our calculation simple, we work with the $\overline{\mathrm{MS}}$ bottomquark mass as input to the bottom-Yukawa coupling as well as to internal propagators. Note that in all cases we choose the masses of the pseudoscalar $A$ and the charged Higgs boson $H^{ \pm}$heavy enough not to open decay modes of the heavy Higgs boson $H$ into them. The corresponding width of the heavy Higgs boson $\Gamma_{H}$ is obtained with $2 \mathrm{HDMC}$. The detailed settings of the scenarios are presented in Table 2.

Scenario $\mathrm{S} 1$ is a standard $2 \mathrm{HDM}$ scenario of type II, similarly to S2. Both have a large value of $\left|s_{\beta-\alpha}\right|$ close to 1 , such that the coupling of the heavy Higgs to gauge bosons proportional to $c_{\beta-\alpha}$ is small. They in particular differ in the choice of the heavy Higgs mass $m_{H}$. Scenario S1 is inspired by the ATLAS analysis carried out in Ref. [58], where we want to discuss the relevance of interferences for the per- 
Table 2 2HDM scenarios considered in our analysis

\begin{tabular}{llrlll}
\hline Scenario & 2HDM type & $t_{\beta}$ & $s_{\beta-\alpha}$ & $m_{H}(\mathrm{GeV})$ & $\Gamma_{H}(\mathrm{GeV})$ \\
\hline S1 & II & 2 & -0.995 & 200 & 0.0277 \\
S2 & II & 1 & 0.990 & 400 & 3.605 \\
S3 & I & 5 & 0.950 & 400 & 2.541 \\
S4 & I & 5 & 0.96695 & 200 & 0.0882 \\
S5 & II & 20 & 0.990 & 400 & 5.120 \\
\hline
\end{tabular}

formed experimental searches in the previous LHC run at $\sqrt{s}=8 \mathrm{TeV}$.

Scenario S3 is of type I, where larger values of $c_{\beta-\alpha}$ are still compatible with data, see Ref. [83]. The last two scenarios S4 and S5 are such that particularly large interferences are possible either between the heavy Higgs boson signal and the light Higgs boson or the background. Potentially large interference effects can occur for values of $s_{\beta-\alpha}$ where the top- (and bottom-quark) Yukawa coupling $g_{t}^{H}$ (and $g_{b}^{H}$ ) for the heavy Higgs boson in a 2HDM of type II (or I) are suppressed. Another possibility is a relatively large value of $t_{\beta}$, which increases the relevance of the bottom-quark Yukawa coupling $g_{b}^{H}$ for the heavy Higgs boson in a 2HDM of type II. A general difference between scenarios S2, S3, and S5 and scenarios S1 and S4 is also the heavy Higgs boson mass. The latter is once above and once below the top threshold $2 m_{t}$. For $m_{H}>2 m_{t}$ on the one hand the decay mode $H \rightarrow t \bar{t}$ opens and on the other hand also an imaginary part of the amplitude $g g \rightarrow H \rightarrow V V$ is induced through the top-quark loop, which is of importance for interferences, see also Ref. [45].

For completeness we also investigated a "flipped Yukawa" scenario, see e.g. Refs. [83-85], where the relative bottom Yukawa coupling of the light Higgs is $g_{b}^{h}=-1$. Such a scenario makes it possible to have a relatively large value for $c_{\beta-\alpha}$ and thus a large coupling of the heavy Higgs boson to gauge bosons keeping the light Higgs compatible with experimental bounds. However, in the processes under consideration it does not provide new features with respect to the other five scenarios and is thus not listed separately.

Our studies are generally carried out for the LHC with a center-of-mass energy of $\sqrt{s}=13 \mathrm{TeV}$, except for scenario $\mathrm{S} 1$ which is investigated both at 8 and $13 \mathrm{TeV}$. The role of interference effects is a bit less pronounced at 7/8 TeV compared to $13 \mathrm{TeV}$. We make use of CT10nnlo [86] as PDF set for the gluon luminosities. Since our calculations are purely performed at LO, the renormalization scale dependence enters through the strong coupling $\alpha_{s}$ only, which we take from the employed PDF set. We choose the renormalization and factorization scale to be dynamical, namely half of the invariant mass of the gauge boson system $\mu_{R}=\mu_{F}=$ $m_{V V} / 2$, i.e. $\mu_{R}=\mu_{F}=m_{4 l} / 2$ in the case of the four leptonic final states. Since the Gram determinants of the box diagrams, see Fig. 1c, approach zero for low $p_{T}$ of the heavy gauge bosons, we perform a technical cut of $p_{T}^{Z}>2 \mathrm{GeV}$ and $p_{T}^{W}>2 \mathrm{GeV}$ for all our processes. It is well known to have a small effect on the cross section [21,25], which we have numerically confirmed for the processes under consideration.

For the processes with four charged leptons or two charged leptons and two neutrinos in the final state, we additionally cut on the transverse momentum and the pseudorapidity of each lepton $l, p_{T}^{l}>10 \mathrm{GeV}$ and $\left|\eta_{l}\right|<2.7$, the $R$-separation between individual leptons $R^{l l^{\prime}}>0.1$, as well as $m_{l l}>$ $5 \mathrm{GeV}$, where $l l$ is an oppositely charged same-flavor dilepton pair. For the neutrinos we ask for a total missing transverse momentum of $E_{T}^{\text {miss }}>70 \mathrm{GeV}$. The cuts are inspired by the recent ATLAS analysis carried out in Ref. [58].

One of the most important observables is certainly the invariant mass distribution of the four leptons, as the two Higgs bosons manifest themselves in Breit-Wigner peaks in this distribution. For the first process $g g \rightarrow e^{+} e^{-} \mu^{+} \mu^{-}$ of Eq. (3) this observable $m_{4 l}$ is also experimentally easily accessible due to two electrons and two muons in the final state. In the cases with neutrinos in the final state the situation is more involved. The invariant mass is no longer an observable that is experimentally accessible but only a transverse component can be measured. As we are particularly interested in a heavy Higgs boson that will decay into the four leptons via two intermediate electroweak gauge bosons, a sensible choice is to consider the transverse mass of the underlying two boson system. In our case the two boson system can be $Z Z$ as well as $W W$. We therefore define a general transverse mass via [87]

$m_{V V, T}^{2}=\left(E_{T, l l}+E_{T, v v}\right)^{2}-\left|\vec{p}_{T, l l}+\vec{p}_{T, v v}\right|^{2}$,

with

$E_{T, l l}=\sqrt{p_{l l}^{2}+\left|\vec{p}_{T, l l}\right|^{2}}$, and $E_{T}^{\mathrm{miss}}=E_{T, v v}=\left|\vec{p}_{T, v v}\right|$.

In the case of a purely leptonic final state the last squared term in Eq. (5) vanishes. As we are interested in the heavy Higgs boson and its interference with the light Higgs boson and the background we put an additional cut on the invariant mass. For the scenarios where the heavy Higgs mass is $400 \mathrm{GeV}$, we require $m_{4 l}>350 \mathrm{GeV}$ for the muonic process. For the 


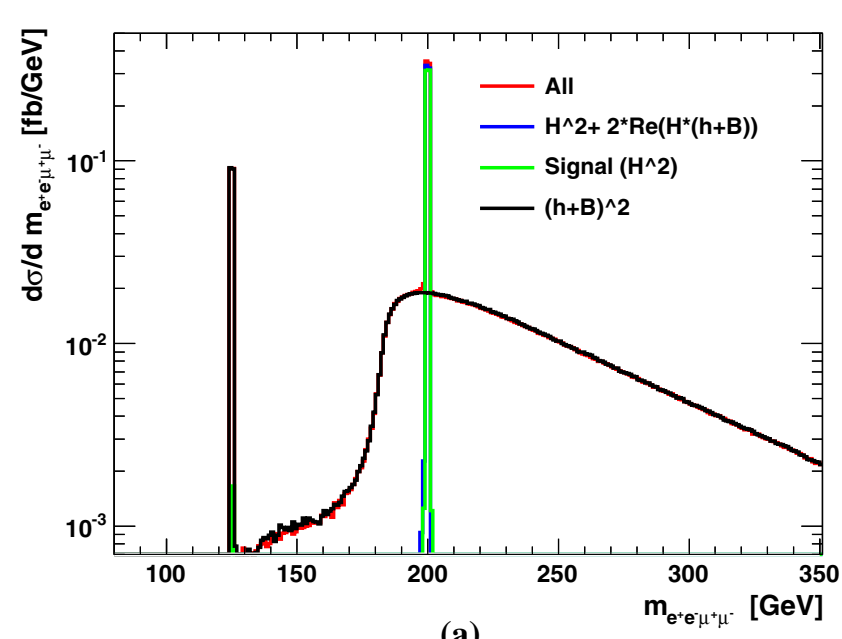

(a)

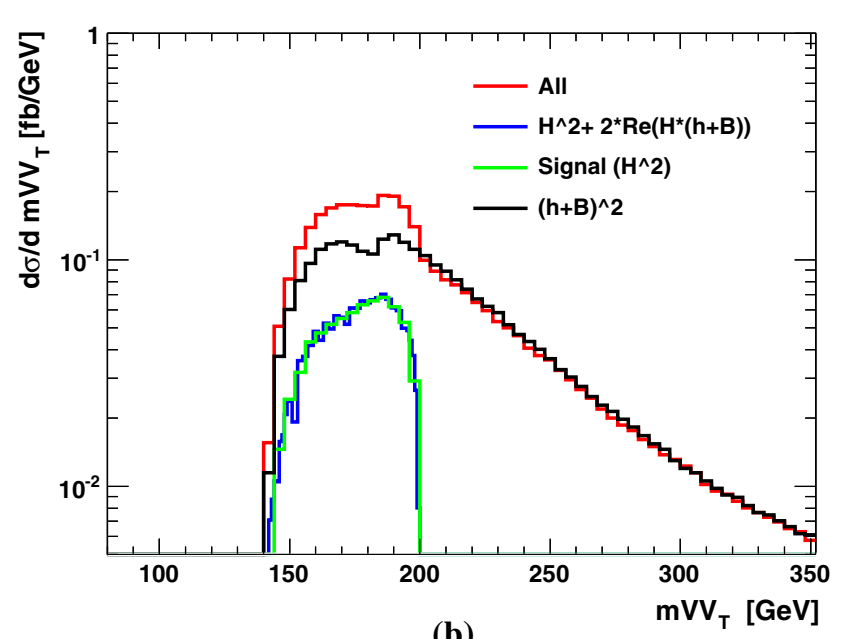

(b)

Fig. 4 a Invariant mass distribution for $g g \rightarrow e^{+} e^{-} \mu^{+} \mu^{-}$and $\mathbf{b}$ transverse mass distribution for $g g \rightarrow e^{+} e^{-} \nu_{l} \bar{\nu}_{l}$ for scenario $\mathrm{S} 1$ at $\sqrt{s}=13 \mathrm{TeV}$

neutrino process we apply the same cut but on $m_{V V, T}$. For the scenarios where the heavy Higgs boson mass is $200 \mathrm{GeV}$, we choose the invariant mass cut as $m_{4 l}>100 \mathrm{GeV}$ or $m_{V V, T}>100 \mathrm{GeV}$, respectively.

\section{Numerical results}

We present our numerical results as follows: We start with a discussion of the four fermionic final states making use of the benchmarks scenarios defined in Sect. 3. Afterwards we exemplify the relevance of interference effects for $g g \rightarrow$ $Z Z$, where we vary either the heavy Higgs mass $m_{H}$, the relative coupling to gauge bosons $s_{\beta-\alpha}\left(c_{\beta-\alpha}\right.$ for the heavy Higgs, respectively) or the ratio of vacuum expectation values $t_{\beta}$ and fix the other parameters according to the benchmark scenarios. The three mentioned free parameters are the ones relevant for the phenomenology of the heavy Higgs boson in the processes under consideration. As noted the heavy Higgs width $\Gamma_{H}$ is obtained from 2 HDMC.

The process $g g \rightarrow V V\left(\rightarrow e^{+} e^{-} \mu^{+} \mu^{-} / e^{+} e^{-} \nu_{l} \bar{\nu}_{l}\right)$ is by far superseded by the large background $q \bar{q} \rightarrow$ $V V\left(\rightarrow e^{+} e^{-} \mu^{+} \mu^{-} / e^{+} e^{-} v_{l} \bar{\nu}_{l}\right)$ (without Higgs boson contributions), in particular at high invariant masses. However, the two processes do not interfere and can be added incoherently. For the relevance of other backgrounds we refer to Ref. [58]. A detailed simulation with all backgrounds in order to determine to which cross sections the experiments are actually sensitive at high integrated luminosities is beyond the scope of this paper. In addition, with increasing $t_{\beta}$ in a $2 \mathrm{HDM}$ of type II the bottom-quark initiated processes $b \bar{b} \rightarrow V V\left(\rightarrow e^{+} e^{-} \mu^{+} \mu^{-} / e^{+} e^{-} \nu_{l} \bar{\nu}_{l}\right)$ become relevant, which involve Higgs boson contributions and thus similar interferences as observed in the gluon initiated processes.

\subsection{Discussion of four-fermion final states}

In the following we discuss the processes

$$
\begin{aligned}
& g g \rightarrow e^{+} e^{-} \mu^{+} \mu^{-}, \quad g g \rightarrow e^{+} e^{-} \nu_{\mu / \tau} \bar{v}_{\mu / \tau}, \\
& \quad g g \rightarrow e^{+} e^{-} \nu_{e} \bar{v}_{e},
\end{aligned}
$$

for the benchmark scenarios and the cuts shown in Sect. 3, taking into account the contributions displayed in Sect. 2.2.

We start the discussion of the numerical results with scenario S1. Figure 4 shows the invariant mass distribution of the four leptons for $g g \rightarrow e^{+} e^{-} \mu^{+} \mu^{-}$and the transverse mass distribution using the definition in Eq. (5) for the processes involving final state neutrinos. In this plot and in the following we distinguish four different contributions. In red, denoted with "All", we plot all contributions that lead to the given final state in the considered scenario. In green, we only plot the contribution from the heavy Higgs boson, whereas in blue we also add the interference of the heavy Higgs boson with the background and the light Higgs boson. The contribution $|h+B|^{2}$, plotted in black, contains besides the contributions without any Higgs also contributions of the light Higgs as well as the interference contributions of the light Higgs boson with non-Higgs diagrams.

In the invariant mass plot of $g g \rightarrow e^{+} e^{-} \mu^{+} \mu^{-}$, see Fig. 4a, the two Higgs boson peaks at $m_{4 l}=125$ and $200 \mathrm{GeV}$ can be clearly seen. Due to the very small width of the heavy Higgs boson there is no distortion of the Breit-Wigner shape visible, and also the impact of the interference contribution to the total height of the peak is rather small. The transverse mass distribution for $g g \rightarrow e^{+} e^{-} v_{l} \bar{\nu}_{l}$, see Fig. 4b, shows a quite different pattern. First of all there is no peak from the light Higgs boson. The reason for this are the different cuts compared to the process without neutrinos. The 


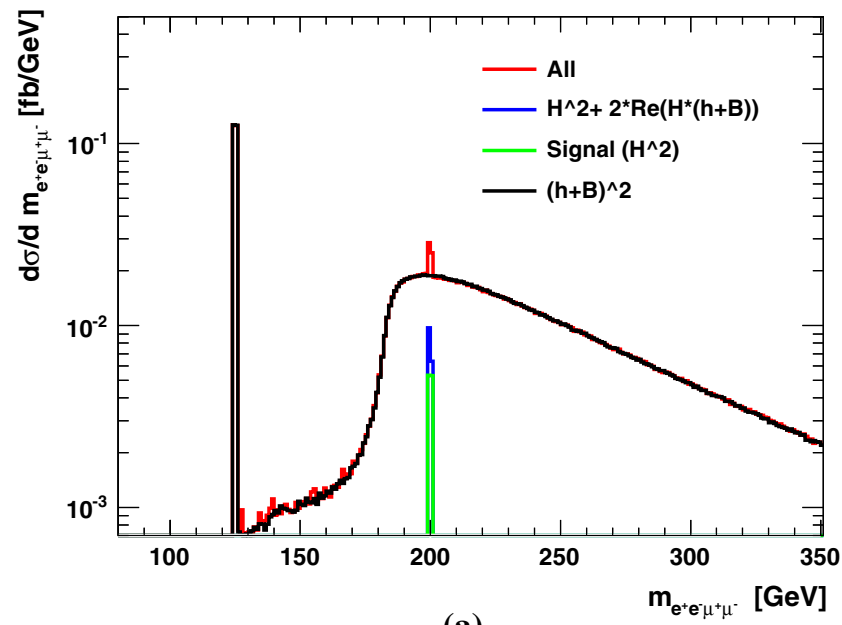

(a)

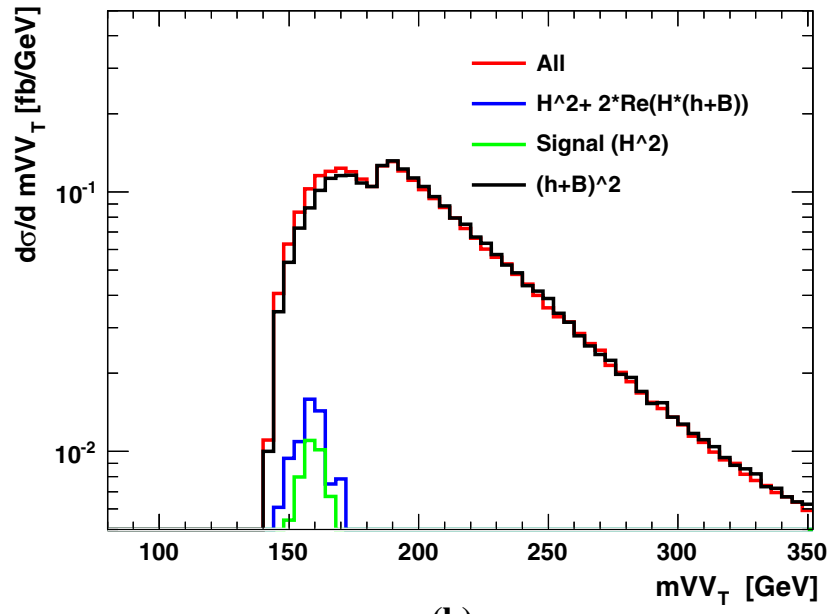

(b)

Fig. 5 a Invariant mass distribution for $g g \rightarrow e^{+} e^{-} \mu^{+} \mu^{-}$and $\mathbf{b}$ transverse mass distribution for $g g \rightarrow e^{+} e^{-} v_{l} \bar{\nu}_{l}$ for scenario $\mathrm{S} 4$ at $\sqrt{s}=13 \mathrm{TeV}$

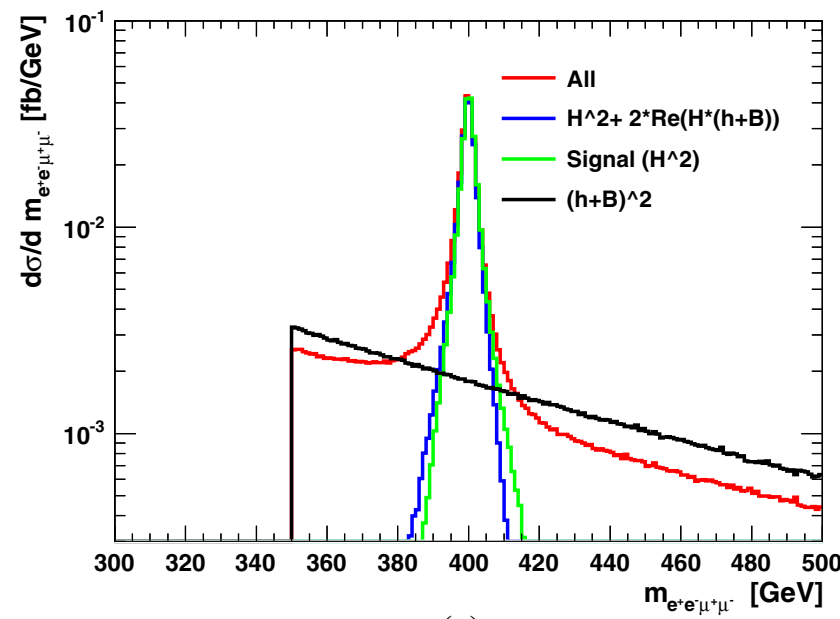

(a)

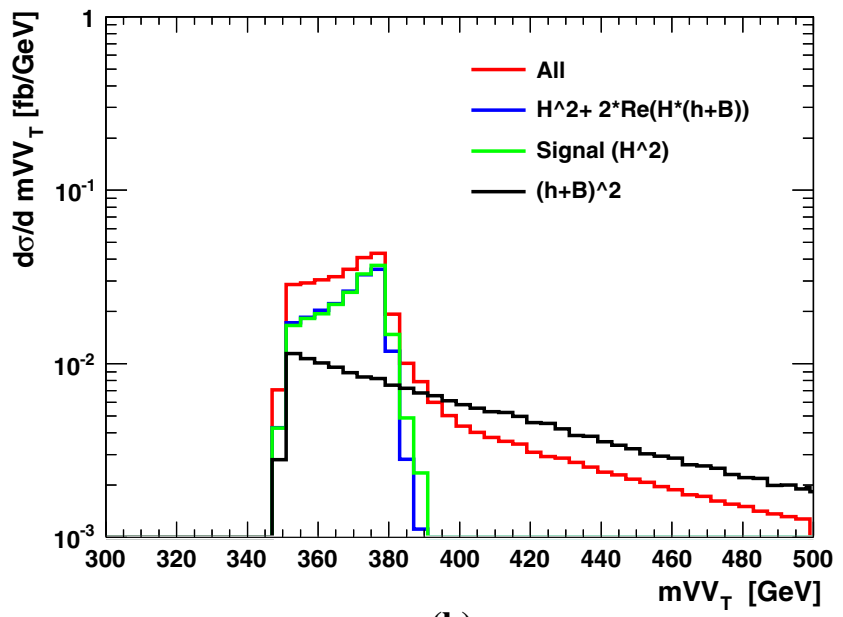

(b)

Fig. 6 a Invariant mass distribution for $g g \rightarrow e^{+} e^{-} \mu^{+} \mu^{-}$and $\mathbf{b}$ transverse mass distribution for $g g \rightarrow e^{+} e^{-} \nu_{l} \bar{\nu}_{l}$ for scenario $\mathrm{S} 2$ at $\sqrt{s}=13 \mathrm{TeV}$

requirement of $E_{T}^{\text {miss }}>70 \mathrm{GeV}$ excludes this region of phase space. Due to the fact that the four-momenta of the neutrinos are experimentally not accessible one sets $E_{T, v v}=\left|\vec{p}_{T, \nu v}\right|$, which ignores the invariant mass of the neutrino system. This removes the sharp peak of the heavy Higgs boson, which is visible in the invariant mass distribution of the muon process. Instead of a distinguished peak one obtains a broad distribution. But also here the contribution of the interference remains small. A second difference compared to the muon process is the occurrence of a small dip at around $m_{V V, T}=180 \mathrm{GeV}$ in both signal and background. This specific shape is due to the fact that the total contribution to the process with neutrino final state consists of the sum of two different subprocesses, namely the one with the electron neutrino and the ones with muon- and tau neutrino in the final state. Whereas the first subprocess also has contributions from intermediate $W$-bosons, this is not the case for the latter subprocesses. The two subprocesses therefore show a different kinematical behavior, and the sum of the two contributions leads to the given distribution.

Scenario S4 shown in Fig. 5 is phenomenologically similar to scenario S1 with a very narrow heavy Higgs boson peak. In contrast to scenario S1 the couplings of the heavy Higgs to the fermions and gauge bosons are smaller leading to a small heavy Higgs boson signal. The relative importance of the interference effect is sizable and increases the heavy Higgs boson signal by roughly a factor of 2 . However, its measurement remains challenging. For the neutrino process on the r.h.s. one observes a more pronounced dip as compared to scenario S1. In contrast to the type I scenario S4, the type II scenario S2 leads to a large signal-over-background ratio for the heavy Higgs peak as can be seen from Fig. 6. Accordingly the interference contribution leads only to a mild distortion of the shape and thus only has a small impact. 


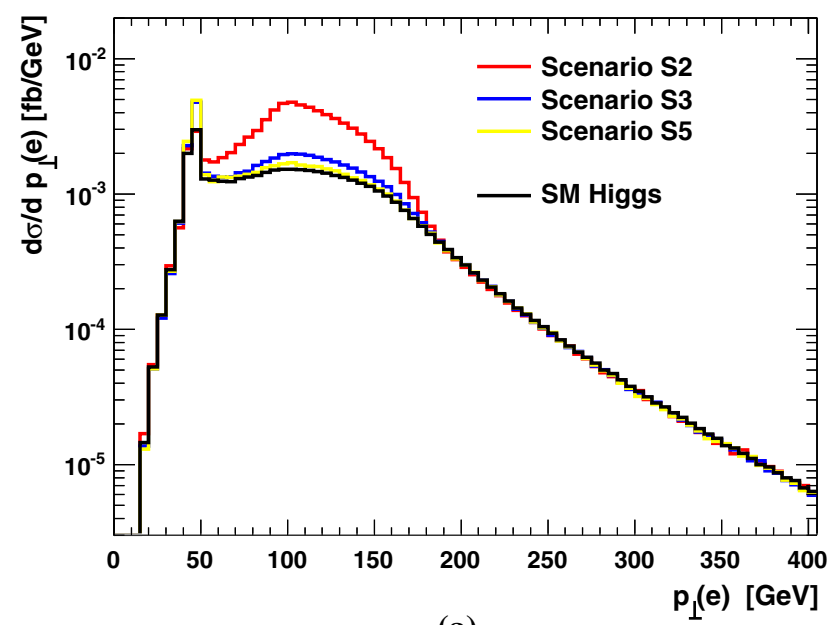

(a)

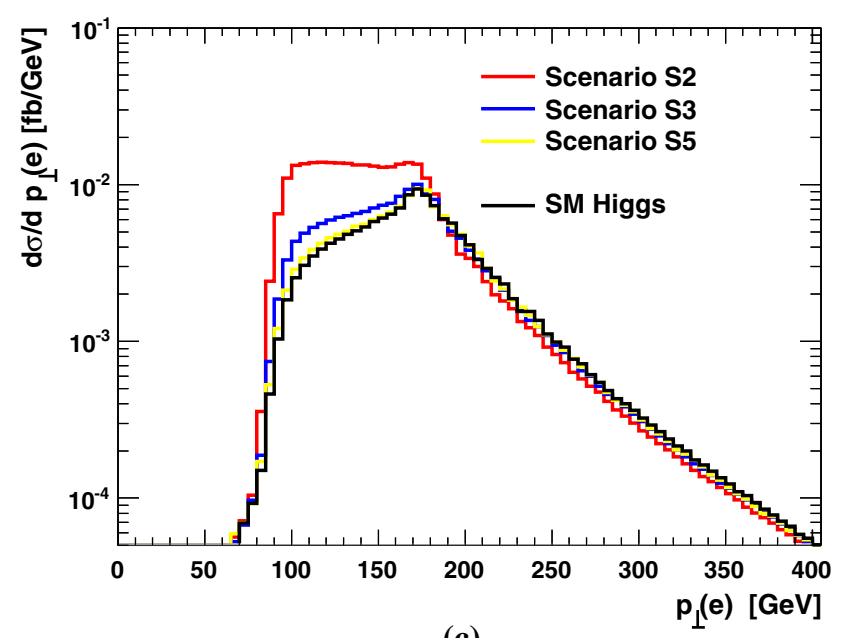

(c)

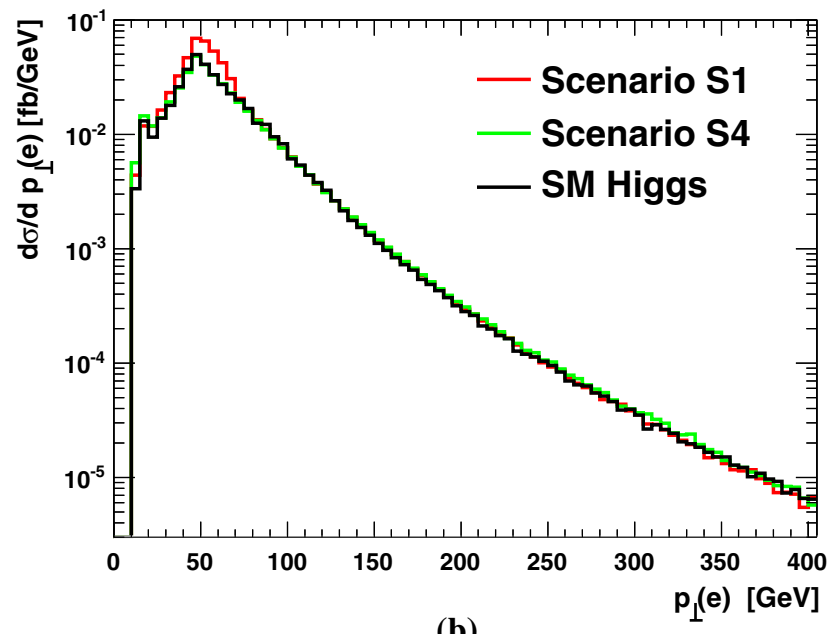

(b)

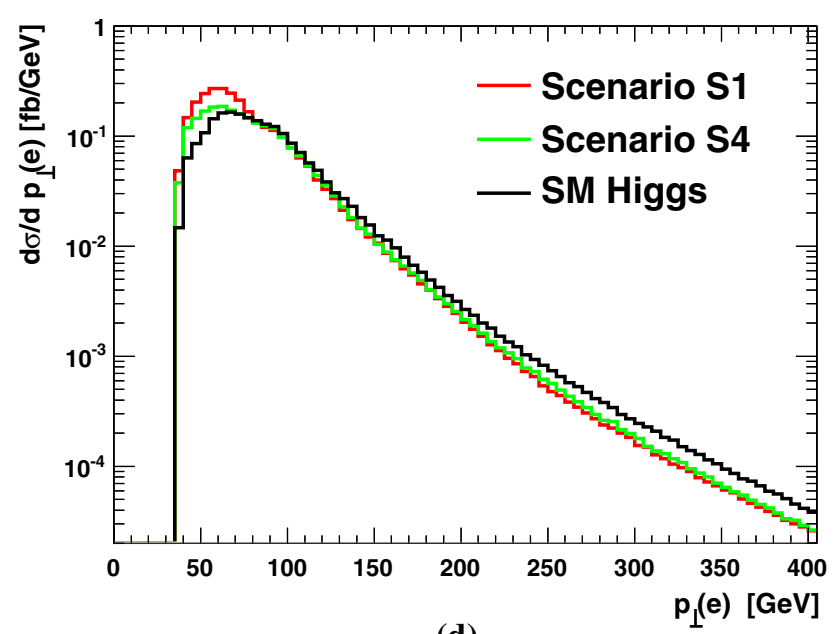

(d)

Fig. 7 Transverse momentum distribution of the hardest electron (electron or positron) for $\mathbf{a}, \mathbf{b} g g \rightarrow e^{+} e^{-} \mu^{+} \mu^{-}$and for $\mathbf{c}, \mathbf{d} g g \rightarrow e^{+} e^{-} \nu_{l} \bar{\nu}_{l}$ at $\sqrt{s}=13 \mathrm{TeV}$. Due to the different cuts on the invariant mass of the four leptons, the scenarios S2, S3 and S5 (a, c) are plotted separately from $\mathrm{S} 1$ and $\mathrm{S} 4(\mathbf{b}, \mathbf{d})$

In general, comparing $g g \rightarrow e^{+} e^{-} \mu^{+} \mu^{-}$with $g g \rightarrow$ $e^{+} e^{-} v_{l} \bar{v}_{l}$, the big advantage of the latter process is the much larger total cross section. We find that for $\sqrt{s}=13 \mathrm{TeV}$ the cross section for the process with neutrinos in the final state is roughly one order of magnitude larger than for $g g \rightarrow e^{+} e^{-} \mu^{+} \mu^{-}$. First there are three neutrino generations involved, second, there are more additional underlying subprocesses (including internal $W$ bosons) and different couplings. On the other hand $g g \rightarrow e^{+} e^{-} \nu_{l} \bar{v}_{l}$ is experimentally more difficult to access than $g g \rightarrow e^{+} e^{-} \mu^{+} \mu^{-}$with four tagged leptons in two different flavors. For the latter process mass windows around the $Z$-boson mass even allow one to cut away non-resonant contributions and only measure the pure $g g \rightarrow Z Z$ contribution.

In the following paragraphs we also discuss how other observables are affected by the presence of a second heavy Higgs boson. In the invariant mass distribution the effect is obvious as the heavy Higgs boson leads to an additional peak in the distribution. In contrast in other observables its presence is less significant but still sizable.

In Fig. 7 we plot the transverse momentum distribution of the hardest electron, being either the involved electron or the positron. In Fig. 7a, b the distribution is plotted for the final state including muons, Fig. 7c, d shows the same distribution for the neutrino final state. The scenarios S2, S3 and $\mathrm{S} 5$ are plotted in a different figure than the scenarios $\mathrm{S} 1$ and $\mathrm{S} 4$ due to the different cuts on the invariant mass of the four leptons (350 and $100 \mathrm{GeV}$ ). In order to investigate the question how the different 2HDM benchmark scenarios can be distinguished from the SM, we also plot the distribution for the SM including the SM Higgs boson. In contrast to the invariant mass and transverse mass spectra we do not split our results into the pure heavy Higgs boson and interference contributions, since the individual contributions 


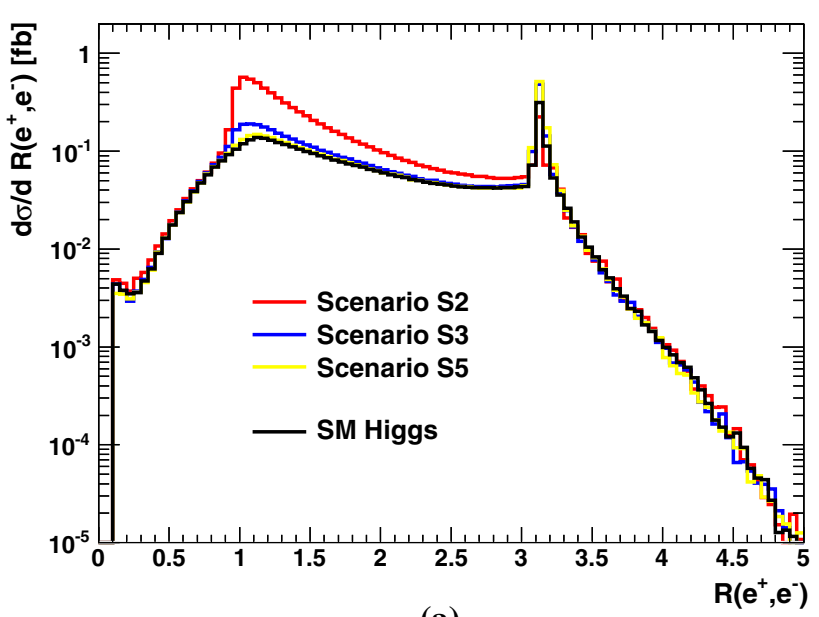

(a)

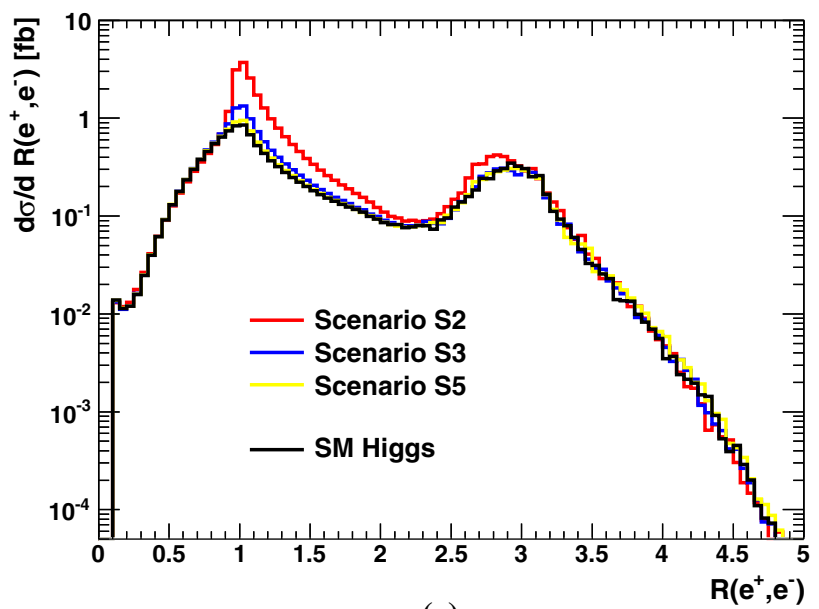

(c)

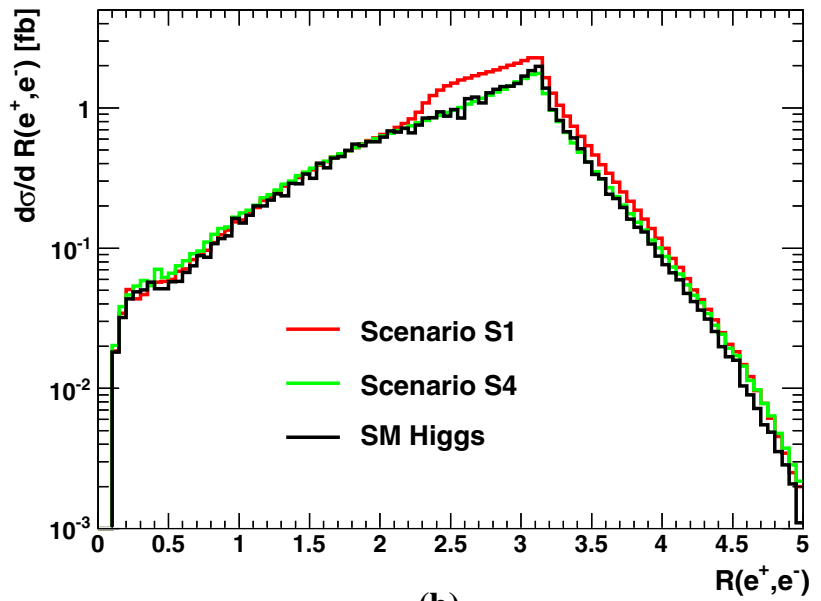

(b)

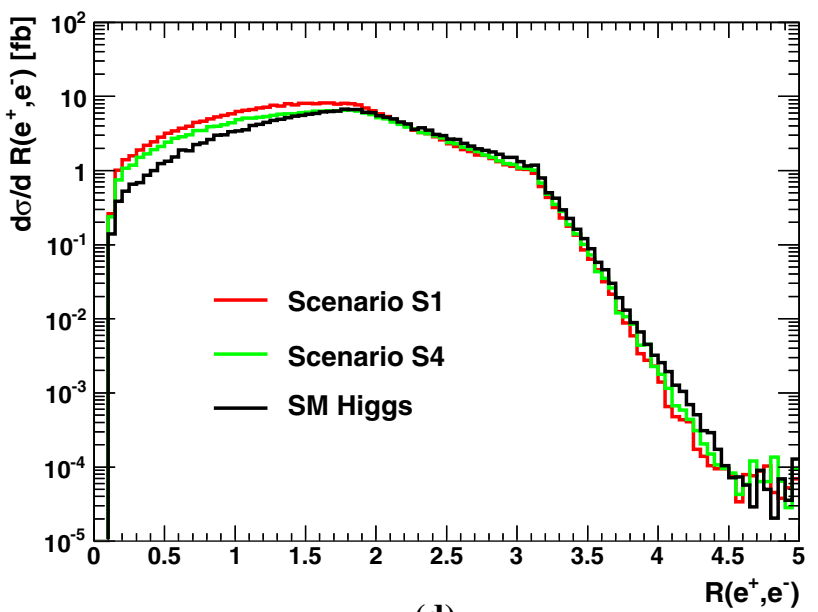

(d)

Fig. $8 R$-separation between electron and positron for $\mathbf{a}, \mathbf{b} g g \rightarrow e^{+} e^{-} \mu^{+} \mu^{-}$and for $\mathbf{c}, \mathbf{d} g g \rightarrow e^{+} e^{-} \nu_{l} \bar{v}_{l}$ at $\sqrt{s}=13 \mathrm{TeV}$. Due to the different cuts on the invariant mass of the four leptons, the scenarios S2, S3, and S5 (a, c) are plotted separately from S1 and S4 (b, d)

would include large invariant mass contributions, not being unitarized.

For the SM with a cut on the invariant mass of $350 \mathrm{GeV}$ we observe a peak coming from the $Z$-boson decay followed by a smoothly falling distribution, see Fig. 7a, c. Reducing the cut to $100 \mathrm{GeV}$ changes the shape of the distribution significantly. In case of the scenarios S2, S3, and S5 we observe a substantial deviation from the Standard Model prediction for the intermediate region $50-200 \mathrm{GeV}$, which is caused by the effects of the additional heavy Higgs boson. For the cases of the scenarios S1 and S4 the effects are much less pronounced. This is partially due to the decreased importance of the heavy Higgs boson but also due to the fact that the heavy Higgs is $200 \mathrm{GeV}$ in these scenarios, which means that the cut on the invariant mass is set to $100 \mathrm{GeV}$. These distributions therefore contain contributions from the light Higgs peak, which makes the heavy Higgs also relatively unimportant. For the neutrino final state one does not observe a peak in the transverse momentum distribution in the low $p_{T}$-region, which can be explained by the additional cut on the missing $E_{T}$ of the neutrinos and by the additional presence of the $W$-pair processes plus the increased importance of off-shell contributions.

Another interesting class of observables are angular correlations. In Fig. 8 we show the $R$-separation between the electron and the positron. Also here we have split the plots according to the different cuts in the same way as described above.

For $g g \rightarrow e^{+} e^{-} \mu^{+} \mu^{-}$we observe a peak of the distribution at $\pi$ which stems from $Z$-boson decays at rest where the leptons are in a back-to-back configuration. Adding an additional heavy Higgs boson leads to $Z$ bosons that are boosted, which means that in the lab frame the distance between the same flavor leptons is reduced. The presence of a heavy Higgs therefore tends to shift the $R$-separation from back-to-back configuration toward smaller values. For the scenarios S1 and S4 this effect is much less pronounced as the importance of the heavy Higgs is reduced and in addition there is 
a contribution from the light Higgs (due to the lower cut on the invariant mass), which, however, cannot lead to boosted $\mathrm{Z}$ bosons. Therefore we do not observe this shift toward smaller values of $R$ but the distribution peaks at $R=\pi$.

For $g g \rightarrow e^{+} e^{-} \nu_{l} \bar{\nu}_{l}$ the peak at $\pi$ is reduced to a kink, again due to the presence of an additional $W$-pair channel, but also here we see the tendency that the presence of the heavy Higgs shifts the $R$-separation toward smaller values.

We note that the presence of a heavy Higgs does not lead to drastic shape distortions of angular correlations and observables of the final state leptons. As both Higgs bosons are scalars, they decay isotropically, which means that the heavy Higgs boson influences rather the kinematics of the leptons than the qualitative decay structure.

\subsection{Relevance of interference contributions exemplified with $g g \rightarrow Z Z$}

In the following study of the relevance of interference contributions we make use of the five benchmark scenarios presented in Table 2 and vary one of the three relevant parameters for our process, namely $m_{H}, \sin (\beta-\alpha)$ and $t_{\beta}$. The combination of the latter two also fixes the coupling of the heavy Higgs to top quarks and bottom quarks, see Eq. (2).

\subsubsection{Dependence on $m_{H}$ in scenarios $S 1$}

In order to make contact with the ATLAS analysis carried out in Ref. [58] we start with a discussion of the interference contributions as a function of $m_{H}$ in scenario S1. In case of S1 the variation within $m_{H}=150-700 \mathrm{GeV}$ corresponds to Fig. 14 of Ref. [58], where we keep $c_{\beta-\alpha}=-0.1$ (in the convention for $\beta-\alpha$ of the ATLAS analysis) fixed (as in Fig. 14 of Ref. [58]) and pick one value of $t_{\beta}$, namely $t_{\beta}=2$, which leads to cross sections close to the achieved experimental sensitivity in the first run of the LHC. To match the numbers of the ATLAS analysis the center-of-mass energy for scenario $\mathrm{S} 1$ is set to $\sqrt{s}=8 \mathrm{TeV}$. We quantify the relevance of the interference as follows: We integrate the differential cross section $\mathrm{d} \sigma^{X} / \mathrm{d} m_{z z}$ for $g g \rightarrow Z Z$ in the range $m_{z z} \in m_{z z}^{I}=\left[m_{H}-15 \mathrm{GeV}, m_{H}+15 \mathrm{GeV}\right]$ and compare the pure heavy Higgs signal peak $X=|H|^{2}$ with the heavy Higgs peak taking into account the interference with the light Higgs $X=|H|^{2}+2 \operatorname{Re}(H \cdot h)$ and the interference with the light Higgs and the background $X=|H|^{2}+2 \operatorname{Re}(H \cdot h)+2 \operatorname{Re}(H \cdot B)$, where the background stems from the box diagrams not involving a Higgs boson. All individual contributions are gauge invariant in this case. The procedure does not take into account the fact that the interferences are often close to be point symmetric with respect to $m_{z z}=m_{H}$, i.e. parts of the interferences below $\left(m_{z z}<m_{H}\right)$ and above $\left(m_{z z}>m_{H}\right)$ the heavy Higgs boson peak cancel each other when integrating over the whole range $m_{z z}^{I}$. This is, however, only of relevance if the peak can be experimentally resolved and of large importance in the case the mass (and possibly width) of a heavy Higgs should be deduced from the peak position and its structure. For a discussion of axially and point symmetric contributions with respect to $m_{z z}=m_{H}$ we also refer to Ref. [45], where the imaginary part of the contributions, due to its axially symmetric structure, is identified to be most relevant for interferences.

Figure 9a shows the Higgs width $\Gamma_{H}$ as a function of the Higgs mass $m_{H}$ for scenario S1. The width $\Gamma_{H}$ increases rapidly above the top-quark threshold $m_{H}>2 m_{t}$, which lowers the inclusive cross section within $m_{z z}^{I}$ significantly, as it can be deduced from Fig. 9b. The latter presents $\sigma^{X}$ for the pure signal $X=|H|^{2}$ in black as well as for $X=|H|^{2}+$ $2 \operatorname{Re}(H \cdot h)$ in red and for $X=|H|^{2}+2 \operatorname{Re}(H \cdot h)+2 \operatorname{Re}(H \cdot B)$ in blue. The relative difference of the contributions with respect to $|H|^{2}$ is shown in Fig. 9c. We deduce that the interference contributions are below $\mathcal{O}(10 \%)$ in the whole parameter range, even in regions where the Higgs width becomes so large that the validity of the narrow-width approximation (NWA) for $H$ can be questioned. Note that the interferences are getting larger for $g g \rightarrow Z Z$, when the Higgs mass approaches the kinematic threshold $2 m_{z}$ from above, since in this example the interferences are point symmetric with respect to $m_{z z}=m_{H}$ and the phase space region $2 m_{z}<m_{z z}<m_{H}$ is reduced. Still we conclude that the usage of the NWA for $H$ in the ATLAS analysis [58] is justified given the obtained sensitivities on the cross sections. Similar conclusions can also be drawn in the mentioned "flipped Yukawa" scenario, even though it allows for slightly larger values of $c_{\beta-\alpha}$.

Given the presented results the width and mass of the heavy Higgs are not the most relevant parameters for what concerns the size of the interferences. As we will see in the two subsequent subsections an increase in the relevance of the interference contributions is observed if the couplings of the heavy Higgs boson, in particular to the top-quark and bottom-quark loop, are suppressed or the latter is significantly enhanced.

\subsubsection{Dependence on $s_{\beta-\alpha}$ in scenarios $S 1$ and $S 4$}

In this subsection we treat $s_{\beta-\alpha}\left(c_{\beta-\alpha}\right)$ as the parameter that is varied and set $t_{\beta}$ to fixed values. For what concerns scenario $\mathrm{S} 1$, this choice corresponds to a line in Fig. 13 of Ref. [58] if we fix $t_{\beta}=2$. Second, we pick the type I scenario $\mathrm{S} 4$. In both cases we vary $c_{\beta-\alpha}^{2}$ in the range $0.00001-1$, which corresponds to $s_{\beta-\alpha}=0-0.999995$. We quantify the interferences as done in the previous subsection again for $\sqrt{s}=8 \mathrm{TeV}$ for S1 and afterwards for $\sqrt{s}=13 \mathrm{TeV}$ for S4.

In Fig. 10a, c, e we present our results for S1. The figures provide complementary information as Fig. 9. Two inter- 
Fig. 9 Scenario $\mathrm{S} 1$ as a function of the heavy Higgs mass $m_{H}$ in $\mathrm{GeV}$ showing a the Higgs width $\Gamma_{H}$ in $\mathrm{GeV}$; b the inclusive cross section $\sigma^{X}$ in pb within $m_{z z}^{I}$ and for $\sqrt{s}=8 \mathrm{TeV}$ (black: $X=|H|^{2}$; red, dashed: $X=|H|^{2}+2 \operatorname{Re}(H \cdot h) ;$ blue, dot-dashed: $X=$ $\left.|H|^{2}+2 \operatorname{Re}(H \cdot h)+2 \operatorname{Re}(H \cdot B)\right)$ c the relative ratio of cross sections $\sigma^{X} / \sigma^{|H|^{2}}$ within $m_{z z}^{I}$
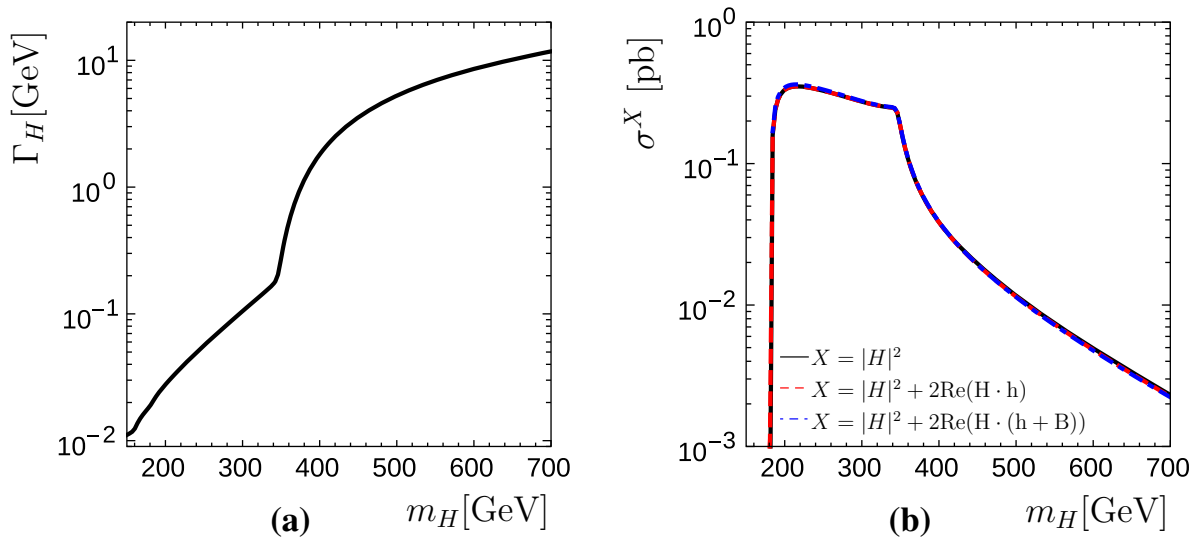

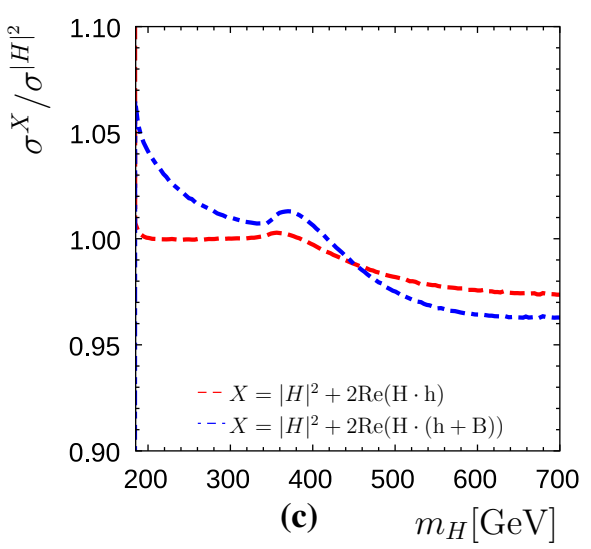

esting observations can be made: The interference is naturally larger in regions where the signal cross section $|H|^{2}$ becomes small as can be seen for very low values of $c_{\beta-\alpha}^{2}$. In addition, and this effect is generic to the 2HDM, the coupling of the heavy Higgs to individual quarks can vanish or the contributions between the top- and bottom-quark cancel. In our example at $c_{\beta-\alpha}^{2}=0.2$ the coupling to top quarks tends to zero, which can easily be understood by decomposing $g_{t}^{H}=\sin \alpha / \sin \beta=-s_{\beta-\alpha} / t_{\beta}+c_{\beta-\alpha}$. For $t_{\beta}>0.7$ the corresponding value of $s_{\beta-\alpha}$, where $g_{t}^{H}=0$, is above $s_{\beta-\alpha}>0.57$ approaching 1 with increasing $t_{\beta}$. Necessarily the interferences in those regions appear to be large. Due to the remaining bottom-quark contributions the minimum is actually located at $c_{\beta-\alpha}^{2}=0.23$ in this specific example. The occurrence of large interferences, however, appears in regions where the signal cross sections is below experimental sensitivities, which reached $\mathcal{O}(0.05-1 \mathrm{pb})$ for the process $g g \rightarrow Z Z$ according to Fig. 12 in Ref. [58] in the first run of the LHC. The observed large effects of interferences occur at lower cross sections, which are, however, potentially of relevance with increasing statistics at the LHC. We show the different distributions $\mathrm{d} \sigma^{X} / \mathrm{d} m_{z z}$ at three values of $c_{\beta-\alpha}$ (marked in Fig. 10c, e) in Fig. 11. Since we obtained the results of Fig. 11 for the partonic cross section and are interested in the relative contributions of the inter- ference effects with respect to the pure heavy Higgs boson signal, we omit units at the $y$-axis. The inclusive cross section can be deduced from Fig. 10c. The three values of $c_{\beta-\alpha}^{2}$ correspond to $s_{\beta-\alpha}=0.999975,0.8888$ and 0.84 .

In Ref. [16] the relevance of interferences for a heavy Higgs boson in $e^{+} e^{-} \rightarrow Z V V / \nu \bar{\nu} V V$ in the context of a $2 \mathrm{HDM}$ at a linear collider was discussed. Since in these processes the Higgs is produced and decays through the couplings to heavy gauge bosons, the coupling $g_{V}^{H}$ occurs twice in each Feynman diagram, whereas $g g \rightarrow Z Z$ only comes with one appearance. However, vector-boson fusion at the LHC also shows a larger suppression of the heavy Higgs boson signal involving the relevant coupling twice. The larger suppression naturally induces larger interference contributions, but, however, small event rates.

We finally discuss in this context a $2 \mathrm{HDM}$ of type I. As we have seen already a crucial quantity is the top-quark Yukawa coupling of the heavy Higgs $g_{t}^{H}$. Since in a 2HDM of type I the relative bottom-quark and top-quark Yukawa coupling are equal, $g_{t}^{H}=g_{b}^{H}$, the cross section as a function of $c_{\beta-\alpha}^{2}$ does indeed vanish for one specific value of $0.57<s_{\beta-\alpha} \leq 1$ rather than just showing a minimum as depicted in Fig. 10c. We show this behavior in Fig. 10b, d, f for scenario S4, which again gives rise to very large interferences in this region, however, with low cross sections below $10^{-1} \mathrm{pb}$. 
Fig. 10 Scenario $S 1(\mathbf{a}, \mathbf{c}, \mathbf{e})$ and scenario $\mathrm{S} 4(\mathbf{b}, \mathbf{d}, \mathbf{f})$ as a function of $c_{\beta-\alpha}^{2}$ showing $\mathbf{a}, \mathbf{b}$ the Higgs width $\Gamma_{H}$ in $\mathrm{GeV} ; \mathbf{c}, \mathbf{d}$ the inclusive cross section $\sigma^{X}$ in pb within $m_{z z}^{I}$ for $\sqrt{s}=8 \mathrm{TeV}$ and $\sqrt{s}=13 \mathrm{TeV}$, respectively (black: $X=|H|^{2}$; red, dashed: $X=|H|^{2}+2 \operatorname{Re}(H \cdot h) ;$ blue, dot-dashed: $X=$

$\left.|H|^{2}+2 \operatorname{Re}(H \cdot h)+2 \operatorname{Re}(H \cdot B)\right)$; $\mathbf{e}, \mathbf{f}$ the relative ratio of cross sections $\sigma^{X} / \sigma^{|H|^{2}}$ within $m_{z z}^{I}$. We show $\mathrm{d} \sigma^{X} / \mathrm{d} m_{z z}$ at the three marked values of $c_{\beta-\alpha}^{2}$ in $\mathbf{c}, \mathbf{e}$ in Fig. 11
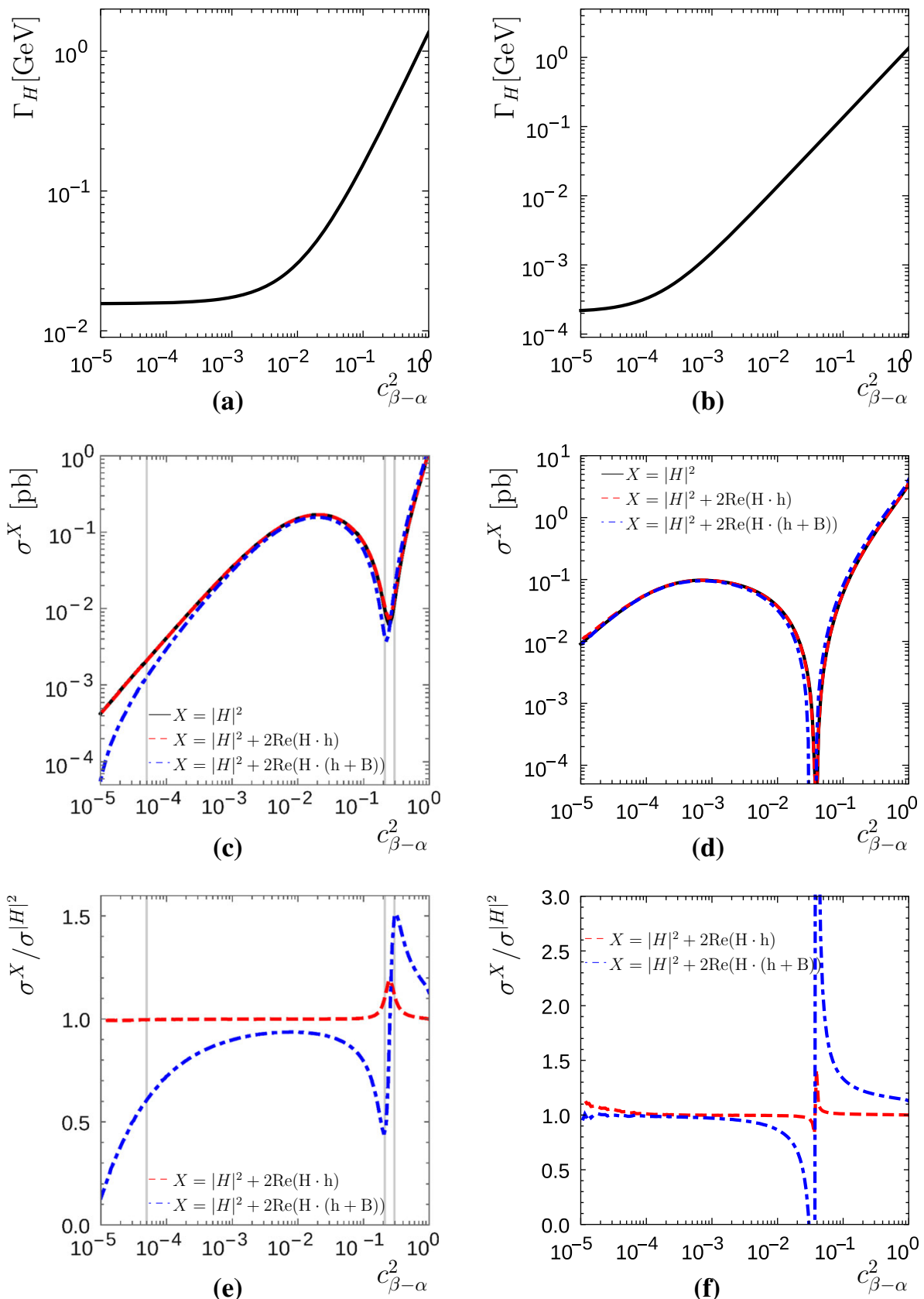

\subsubsection{Dependence on $t_{\beta}$ in $S 2$}

Lastly we focus on the dependence on $t_{\beta}$ in a 2HDM type II, where with increasing $t_{\beta}$ the coupling to bottom-quarks tends to be enhanced for all Higgs bosons. Figure 12 shows the Higgs width $\Gamma_{H}$ and the relevance of the interferences as a function of $t_{\beta}$ for scenario S2. With increasing $t_{\beta}$ the total width $\Gamma_{H}$, depicted in Fig. 12a, first drops due to the drop in the partial width $H \rightarrow t \bar{t}$, for high $\tan \beta$ it rises due to the increase of the partial width $H \rightarrow b \bar{b}$. A fraction of heavy Higgs bosons is also decaying into a pair of lighter Higgs bosons $H \rightarrow h h$. Since, however, the latter decay mode depends on the values of $m_{A}$ and $m_{H}^{ \pm}$indirectly through the parameters of the Higgs potential, we stop at $t_{\beta}=20$ where $H \rightarrow h h$ is still sub-dominant and our results can be considered to a large extent independent of $m_{A}, m_{H^{ \pm}}$and $m_{12}^{2}$. We show the inclusive cross section again within the interval $m_{z z}^{I}=\left[m_{H}-15 \mathrm{GeV}, m_{H}+15 \mathrm{GeV}\right]$ in Fig. $12 \mathrm{~b}, \mathrm{c}$ for $\sqrt{s}=13 \mathrm{TeV}$. The local minimum in Fig. $12 \mathrm{~b}$ at $t_{\beta} \sim 7$ again corresponds to $g_{t}^{H}=0$, which also explains the short rise of the cross section for $t_{\beta}>7$. With increasing $t_{\beta}$ the cross section $\sigma^{|H|^{2}}$ then constantly drops, the interferences on the other hand quickly gain in size. In addition, we show the form of the interferences for $t_{\beta}=20$ in Fig. 12d, which 


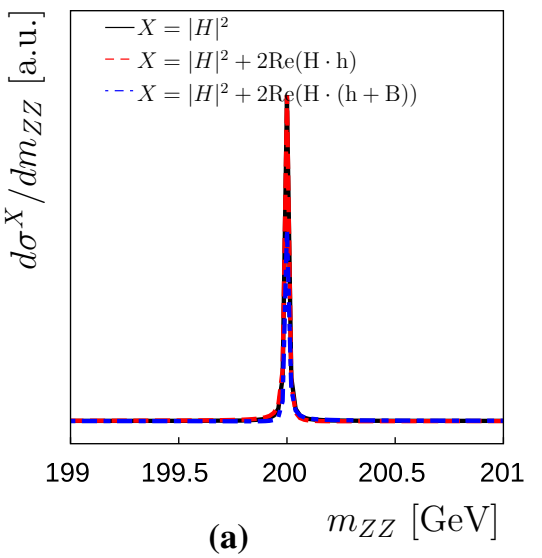

(a)

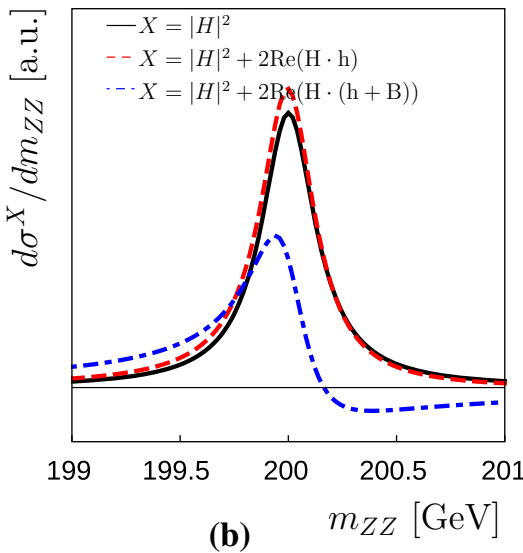

(b)

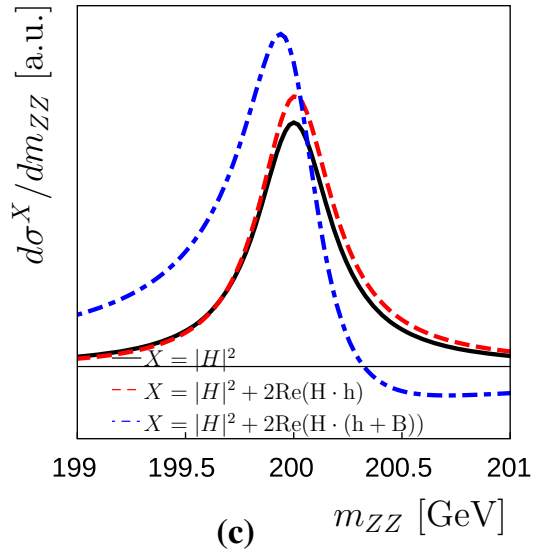

(c)
Fig. 11 Partonic cross sections $\mathrm{d} \sigma^{X} / \mathrm{d} m_{z z}$ in arbitrary units (see text) as a function of $m_{z z}$ in $\mathrm{GeV}$ for $\mathrm{S} 1$ for three different values of $c_{\beta-\alpha}^{2}$ marked in Fig. 10c, e, namely a $c_{\beta-\alpha}^{2}=0.00005 ; \mathbf{b} c_{\beta-\alpha}^{2}=0.21$ and $\mathbf{c} c_{\beta-\alpha}^{2}=0.2944$. The color coding again corresponds to black: $X=|H|^{2} ;$ red, dashed: $X=|H|^{2}+2 \operatorname{Re}(H \cdot h)$; blue, dot-dashed: $X=|H|^{2}+2 \operatorname{Re}(H \cdot h)+2 \operatorname{Re}(H \cdot B)$
Fig. 12 Scenario S2 (S5) as a function of $t_{\beta}$ showing a the Higgs width $\Gamma_{H}$ in $\mathrm{GeV}$; $\mathbf{b}$ the inclusive cross section $\sigma^{X}$ in pb within $m_{z z}^{I}$ for $\sqrt{s}=13 \mathrm{TeV}$ (black: $X=|H|^{2}$; red, dashed: $X=|H|^{2}+2 \operatorname{Re}(H \cdot h) ;$ blue, dot-dashed: $X=$ $\left.|H|^{2}+2 \operatorname{Re}(H \cdot h)+2 \operatorname{Re}(H \cdot B)\right)$ c the relative ratio of cross sections $\sigma^{X} / \sigma^{|H|^{2}}$ within $m_{z z}^{I}$. The partonic cross section $\mathrm{d} \sigma^{X} / \mathrm{d} m_{z z}$ in arbitrary units (see text) is shown in $\mathbf{d}$ as a function of $m_{z z}$ in $\mathrm{GeV}$ for scenario S5 (S2 with $t_{\beta}=20$ )
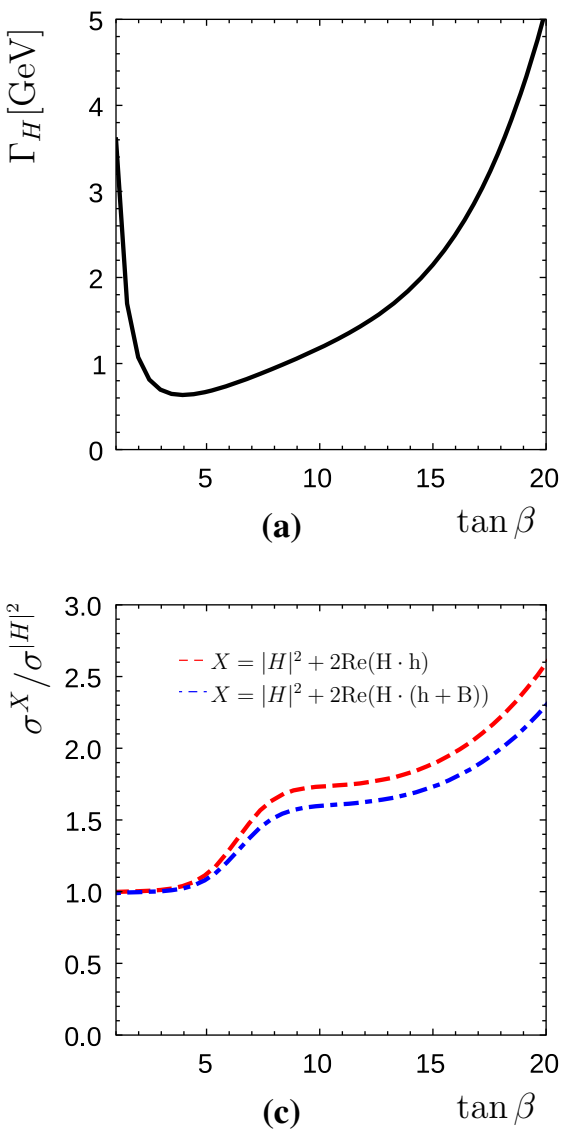
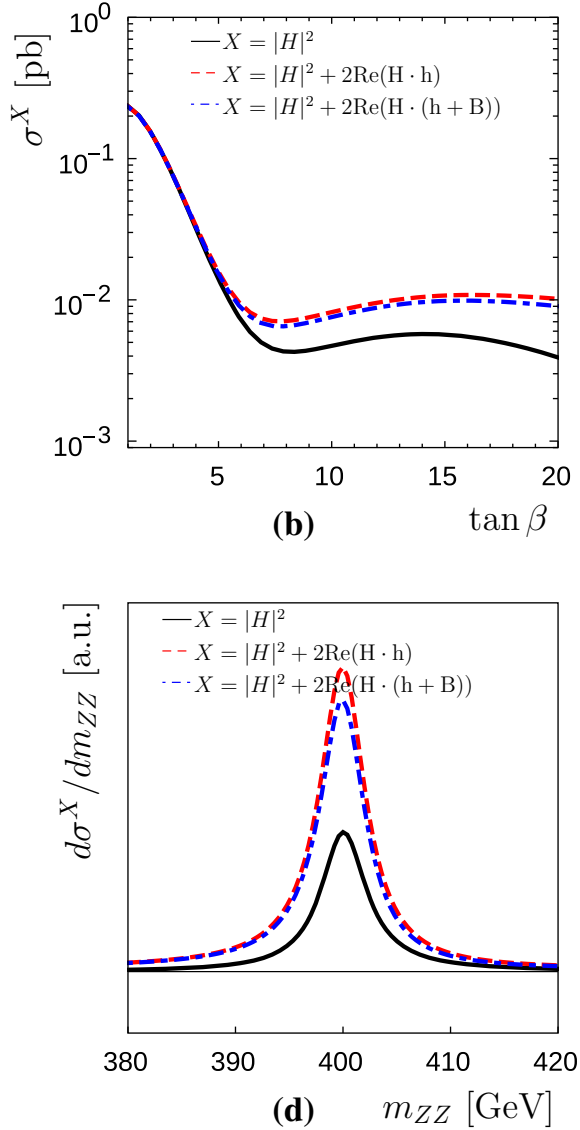

defines scenario S5. Again these large interferences occur below the current experimental sensitivity, but being above $10^{-2} \mathrm{pb}$ the region is potentially in reach for high statistics at the LHC. We note that values of $t_{\beta}>2$ in combination with $s_{\beta-\alpha}=0.99$ for S2 are meanwhile excluded from the non-compatibility with the light Higgs boson signal, see e.g. Ref. [83]. However, the qualitative features are the same in phenomenologically viable scenarios with larger values of $s_{\beta-\alpha}$, as we have numerically checked for $s_{\beta-\alpha}=0.999$.

The interference contribution with the light Higgs boson in this specific case is always positive both below and above the heavy Higgs mass peak $m_{z z}=m_{H}$, see Fig. 12d, and significantly larger than in the previously discussed scenarios due to the large bottom-quark contribution to $g g \rightarrow H \rightarrow Z Z$, 
Fig. 13 Partonic cross sections $\mathrm{d} \sigma^{X} / \mathrm{d} m_{z z}$ as a function of the invariant mass $m_{z z}$ in $\mathrm{GeV}$ for scenario a S2, b S3 and c S5 (black: $X=|H|^{2}$; red, dashed: $X=|H|^{2}+2 \operatorname{Re}(H \cdot h) ;$ blue, dot-dashed: $X=$ $|H|^{2}+2 \operatorname{Re}(H \cdot h)+2 \operatorname{Re}(H \cdot B)$; green, dotted:

$X=|H|^{2}+2 \operatorname{Re}(H \cdot h)+$ $2 \operatorname{Re}(H \cdot B)+2 \operatorname{Re}(h \cdot B))$
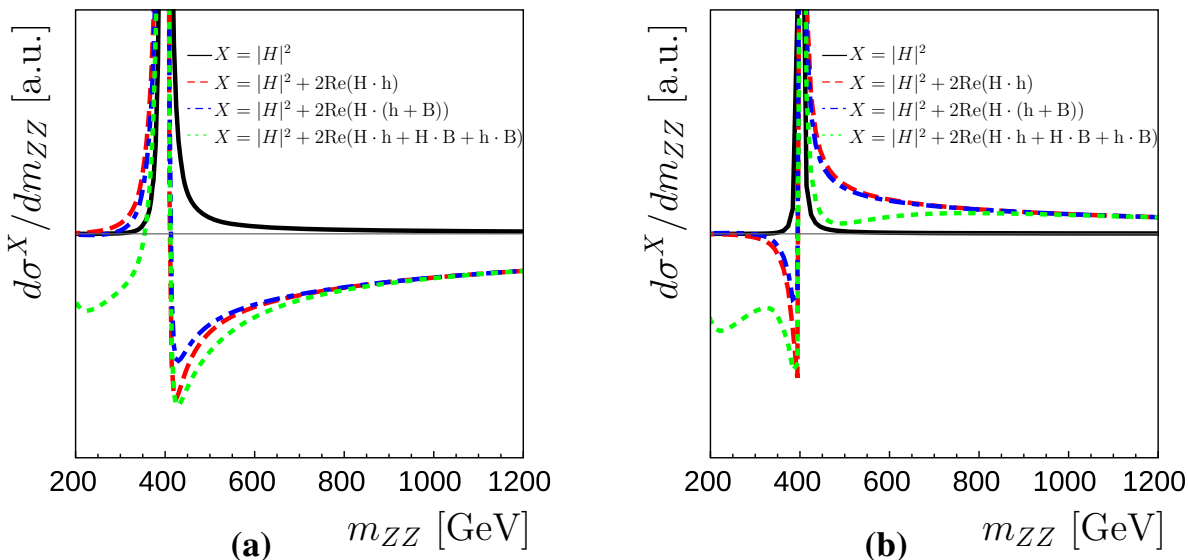

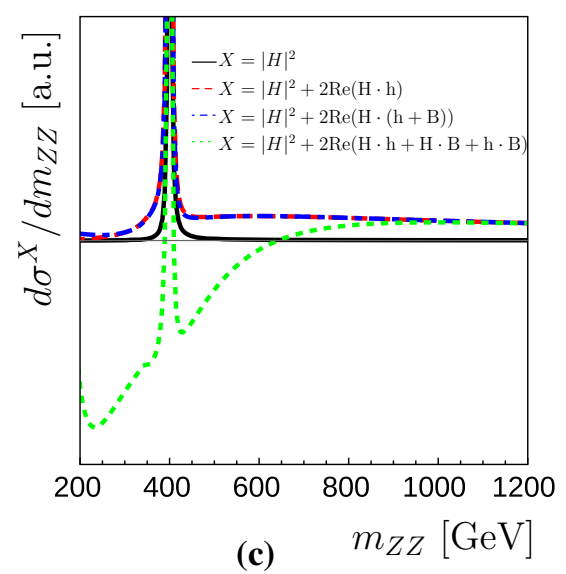

which interferes with the bottom- and top-quark contribution to $g g \rightarrow h \rightarrow Z Z$. In contrast the interference with the background yields a much smaller and negative contribution to the inclusive cross section within $m_{z z}^{I}$, which reflects the unitarization of the cross section for large $m_{z z}$. Increasing $s_{\beta-\alpha}$ to 0.999 yields a similar picture, where the large positive interference is completely dominated by the interference of the bottom-quark contribution to $g g \rightarrow H \rightarrow Z Z$ with the top-quark contribution to $g g \rightarrow h \rightarrow Z Z$, however, the total inclusive cross section is reduced to values slightly below $10^{-2} \mathrm{pb}$. Reference [45] did not point out the relevance of the interference of the heavy Higgs signal with the light Higgs signal for large values of $\tan \beta$, however, emphasized the importance of the bottom-quark loop in $g g \rightarrow H \rightarrow Z Z$ for what concerns the interference with the background. Lastly we comment on the influence of the heavy Higgs boson mass. Below the threshold of the $H \rightarrow h h$ decays, i.e. $m_{H}<250 \mathrm{GeV}$, the size of interference with the light Higgs in $g g \rightarrow Z Z$ is also diminished due to the increase of $\sigma^{|H|^{2}}$. The negative interference with the background gets sizable and reduces the cross section by about $50 \%$. Above $m_{H}>2 m_{h}$, however, $\sigma^{X} / \sigma^{|H|^{2}}$ always significantly differs from 1 . The interferences in these regions can thus significantly enhance the sensitivity to the heavy Higgs boson in experimental searches.
In total we conclude that in particular for large values of $t_{\beta}$ or vanishing $g_{t}^{H}$ interferences can get of importance for future experimental analyses. In the first case the interference of the heavy Higgs contribution with the light Higgs can be significantly enhanced, in the second case the interference with the background. Those cases appear in regions where the inclusive cross sections are in the vicinity of $10^{-2} \mathrm{pb}$ and thus potentially in reach with higher statistics at the LHC.

\subsubsection{Interferences at high invariant masses}

So far we focused on the interference effects between the heavy Higgs and the background as well as the heavy Higgs and the light Higgs in the vicinity of the heavy Higgs resonance. Within the region of the heavy Higgs mass peak the interference between the light Higgs and the background can be considered constant, with a negative contribution to $\mathrm{d} \sigma / \mathrm{d} m_{z z}$, and was therefore not considered in the discussion of $g g \rightarrow Z Z$ so far. However, similar to our discussion of the processes with four fermionic final states we now add the interference of the light Higgs boson with background diagrams also to $g g \rightarrow Z Z$, since at high invariant masses the interplay between all three contributions, $h$ and $H$, and the background $B$, is of relevance and plays a role in the unita- 
rization of the cross section. In Fig. 13 we plot the differential cross section as a function of the invariant mass of the diboson system up to high masses beyond the heavy Higgs resonance. We exemplify the discussion for the three scenarios S2, S3, and S5. The differences between the colored curves display the importance of the different interference terms. Since again our study is performed for the partonic cross section and we are interested in the relative effects of the interferences among each other, we do not display units for $\mathrm{d} \sigma / \mathrm{d} m_{z z}$. At high invariant masses the interference between the heavy Higgs boson and the background is negligible, in contrast to the interference of the light Higgs and the heavy Higgs boson, which can be large and can have either sign. Moreover, the interference of the light Higgs boson and the background has a sizable impact below and above $m_{z z}=m_{H}$ up to invariant masses of about $1 \mathrm{TeV}$. It should be noted that the interference effects below $m_{z z}=m_{H}$ are not easy to distinguish from the larger backgrounds in this region and are also reduced by our selection cuts. In the decoupling limit $\left|s_{\beta-\alpha}\right| \rightarrow 1$ the interference contributions of the light Higgs boson are of course larger than the interference $H \cdot B$. Figure 13 depicts different cases where the interference $h \cdot H$ is either negative, similar to the interference $h \cdot B$, or leads to a positive contribution to the differential cross section in a region $m_{z z} \in[450 \mathrm{GeV}, 1000 \mathrm{GeV}]$. The latter case is realized for scenarios S3 and S5, where the sum of the contributions entering with different sign gives rise to a "peak"-like structure. This structure also appears in the total four particle final state, where the gluon luminosities further suppress the cross section at high invariant masses. As a consequence, all interferences need to be taken into account in order to properly model "peak"-like structures of this kind and thus to correctly describe the cross section at high invariant masses.

\section{Conclusions}

We have investigated the production of a heavy Higgs boson of a $\mathcal{C P}$-conserving Two-Higgs-Doublet-Model in gluon fusion and its subsequent decay into a four-fermion final state. We have discussed in this context the invariant mass and transverse mass distributions for $g g \rightarrow e^{+} e^{-} \mu^{+} \mu^{-}$ and $g g \rightarrow e^{+} e^{-} \nu_{l} \bar{\nu}_{l}$, respectively, as well as other kinematical observables like the separation between outgoing same-flavor leptons and the transverse momentum distributions of the hardest electron/positron. The analysis has been carried out for five different benchmark scenarios. The relative importance of the interference contributions between the heavy Higgs boson, the light Higgs boson, and the background has been investigated for the process in the on-shell approximation, $g g \rightarrow Z Z$. The employed code, GoSam, makes it possible to consistently take into account all mentioned interferences for the four-fermion final states, which should be of interest for future heavy Higgs boson searches.

We have shown that the interference effects are essential for a correct description of the differential cross section, in particular at high invariant masses of the gauge boson system. Rather than being a trivial function of the heavy Higgs mass $m_{H}$ and the heavy Higgs width $\Gamma_{H}$, the mentioned interferences are in particular of relevance in regions of the parameter space where the heavy Higgs boson signal is diminished by small couplings. In case of a 2HDM of type II the enhancement of the bottom-Yukawa coupling for large values of $\tan \beta$ can also significantly enlarge the interference effects. We have investigated the approximation made in the recent ATLAS analysis in the 2HDM to neglect the interference contributions of the heavy Higgs boson with the background and the light Higgs boson. We have found that the relative importance of those interference contributions is at the level of $\mathcal{O}(10 \%)$ with respect to the heavy Higgs boson signal cross section, and that the approximation to neglect the interference contributions involving the heavy Higgs boson was indeed justified in view of the experimental sensitivity that has been reached in the first run of the LHC. We have pointed out, however, that regions of the differential cross sections where interference effects are much larger are potentially in reach at high integrated luminosities. As an important result in this context we have found that interference contributions can significantly enhance the sensitivity to the heavy Higgs boson in experimental searches. In the vicinity of the heavy Higgs boson resonance the interference contributions are particularly important, since they simultaneously alter the form and the position of the heavy Higgs boson mass peak. We have furthermore pointed out that the interference $h \cdot H$, which can enter with either sign, in combination with other contributions can actually mimic a "peak"like structure. An accurate modeling of effects of this kind, which requires the proper incorporation of all interference contributions, is clearly highly relevant for future searches at high invariant masses.

Acknowledgments The authors acknowledge support by "Deutsche Forschungsgemeinschaft" through the SFB 676 "Particles, Strings and the Early Universe". This research was supported in part by the European Commission through the "HiggsTools" Initial Training Network PITN-GA-2012-316704. NG was supported by the Swiss National Science Foundation under contract PZ00P2_154829. We acknowledge the DESY Bird/NAF Cluster and the Helmholtz Alliance "Physics at the Terascale" for providing resources for parts of our numerical results.

Open Access This article is distributed under the terms of the Creative Commons Attribution 4.0 International License (http://creativecomm ons.org/licenses/by/4.0/), which permits unrestricted use, distribution, and reproduction in any medium, provided you give appropriate credit to the original author(s) and the source, provide a link to the Creative Commons license, and indicate if changes were made.

Funded by $\mathrm{SCOAP}^{3}$. 


\section{References}

1. G. Aad et al. (ATLAS Collaboration), Phys. Lett. B 716, 1 (2012). arXiv: 1207.7214

2. S. Chatrchyan et al. (CMS Collaboration), Phys. Lett. B 716, 30 (2012). arXiv:1207.7235

3. H.M. Georgi, S.L. Glashow, M.E. Machacek, D.V. Nanopoulos, Phys. Rev. Lett. 40, 692 (1978)

4. S. Dawson, Nucl. Phys. B 359, 283 (1991)

5. A. Djouadi, M. Spira, P.M. Zerwas, Phys. Lett. B 264, 440 (1991)

6. D. Graudenz, M. Spira, P.M. Zerwas, Phys. Rev. Lett. 70, 1372 (1993)

7. M. Spira, A. Djouadi, D. Graudenz, P.M. Zerwas, Nucl. Phys. B 453, 17 (1995). arXiv:hep-ph/9504378

8. R.V. Harlander, W.B. Kilgore, Phys. Rev. Lett. 88, 201801 (2002). arXiv:hep-ph/0201206

9. C. Anastasiou, K. Melnikov, Nucl. Phys. B 646, 220 (2002). arXiv:hep-ph/0207004

10. V. Ravindran, J. Smith, W.L. van Neerven, Nucl. Phys. B 665, 325 (2003). arXiv:hep-ph/0302135

11. C. Anastasiou, C. Duhr, F. Dulat, F. Herzog, B. Mistlberger, Phys. Rev. Lett. 114(21), 212001 (2015). arXiv:1503.06056

12. S. Dittmaier et al. (LHC Higgs Cross Section Working Group Coll.), arXiv: 1101.0593

13. S. Dittmaier et al. (LHC Higgs Cross Section Working Group Coll.), arXiv:1201.3084

14. S. Heinemeyer et al. (LHC Higgs Cross Section Working Group Coll.), arXiv: 1307.1347

15. N. Kauer, G. Passarino, JHEP 1208, 116 (2012). arXiv: 1206.4803

16. S. Liebler, G. Moortgat-Pick, G. Weiglein, JHEP 1506, 093 (2015). arXiv: 1502.07970

17. E.W.N. Glover, J.J. van der Bij, Phys. Lett. B 219, 488 (1989)

18. E.W.N. Glover, J.J. van der Bij, Nucl. Phys. B 321, 561 (1989)

19. T. Binoth, M. Ciccolini, N. Kauer, M. Kramer, JHEP 0612, 046 (2006). arXiv:hep-ph/0611170

20. J.M. Campbell, R.K. Ellis, C. Williams, JHEP 1110, 005 (2011). arXiv: 1107.5569

21. N. Kauer, PoS RADCOR 2011, 027 (2011). arXiv:1201.1667

22. G. Passarino, JHEP 1208, 146 (2012). arXiv:1206.3824

23. F. Cascioli, S. Höche, F. Krauss, P. Maierhöfer, S. Pozzorini, F. Siegert, JHEP 1401, 046 (2014). arXiv: 1309.0500

24. N. Kauer, JHEP 1312, 082 (2013). arXiv:1310.7011

25. J.M. Campbell, R.K. Ellis, C. Williams, JHEP 1404, 060 (2014). arXiv: 1311.3589

26. J.M. Campbell, R.K. Ellis, C. Williams, PoS LL 2014, 008 (2014). arXiv: 1408.1723

27. F. Campanario, Q. Li, M. Rauch, M. Spira, JHEP 1306, 069 (2013). arXiv: 1211.5429

28. J.M. Campbell, R.K. Ellis, E. Furlan, R. Röntsch, Phys. Rev. D 90(9), 093008 (2014). arXiv:1409.1897

29. M. Bonvini, F. Caola, S. Forte, K. Melnikov, G. Ridolfi, Phys. Rev. D 88(3), 034032 (2013). arXiv: 1304.3053

30. C.S. Li, H.T. Li, D.Y. Shao, J. Wang, JHEP 1508, 065 (2015). arXiv: 1504.02388

31. N. Kauer, C. O’Brien, E. Vryonidou, JHEP 1510, 074 (2015). arXiv:1506.01694

32. S. Dawson, S. Dittmaier, M. Spira, Phys. Rev. D 58, 115012 (1998). arXiv:hep-ph/9805244

33. L. Altenkamp, S. Dittmaier, R.V. Harlander, H. Rzehak, T.J.E. Zirke, JHEP 1302, 078 (2013). arXiv: 1211.5015

34. Z. Bern, L.J. Dixon, C. Schmidt, Phys. Rev. D 66, 074018 (2002). arXiv:hep-ph/0206194

35. F. Caola, J.M. Henn, K. Melnikov, A.V. Smirnov, V.A. Smirnov, JHEP 1506, 129 (2015). arXiv:1503.08759
36. A. von Manteuffel, L. Tancredi, JHEP 1506, 197 (2015). arXiv: 1503.08835

37. K. Melnikov, M. Dowling, Phys. Lett. B 744, 43 (2015). arXiv:1503.01274

38. F. Caola, K. Melnikov, R. Röntsch, L. Tancredi, Phys. Lett. B 754, 275 (2016). doi:10.1016/j.physletb.2016.01.046

39. J.F. Gunion, H.E. Haber, G.L. Kane, S. Dawson, Front. Phys. 80, $1(2000)$

40. A.G. Akeroyd, Phys. Lett. B 377, 95 (1996). arXiv:hep-ph/9603445

41. A.G. Akeroyd, J. Phys. G 24, 1983 (1998). arXiv:hep-ph/9803324

42. M. Aoki, S. Kanemura, K. Tsumura, K. Yagyu, Phys. Rev. D 80, 015017 (2009). arXiv:0902.4665

43. G.C. Branco, P.M. Ferreira, L. Lavoura, M.N. Rebelo, M. Sher, J.P. Silva, Phys. Rep. 516, 1 (2012). arXiv:1106.0034

44. N. Craig, S. Thomas, JHEP 1211, 083 (2012). arXiv:1207.4835

45. S. Jung, J. Song, Y.W. Yoon, arXiv: 1510.03450

46. S. Jung, J. Song, Y.W. Yoon, Phys. Rev. D 92(5), 055009 (2015). arXiv:1505.00291

47. B. Hespel, D. Lopez-Val, E. Vryonidou, JHEP 1409, 124 (2014). arXiv: 1407.0281

48. B. Hespel, F. Maltoni, E. Vryonidou, JHEP 1506, 065 (2015). arXiv:1503.01656

49. E. Maina, JHEP 1506, 004 (2015). arXiv:1501.02139

50. N. Kauer, C. O’Brien, Eur. Phys. J. C 75, 374 (2015). arXiv: 1502.04113

51. A. Ballestrero, E. Maina, JHEP 1601, 045 (2016). doi:10.1007/ JHEP01(2016)045

52. G. Cullen, N. Greiner, G. Heinrich, G. Luisoni, P. Mastrolia, G. Ossola, T. Reiter, F. Tramontano, Eur. Phys. J. C 72, 1889 (2012). arXiv: 1111.2034

53. G. Cullen, H. van Deurzen, N. Greiner, G. Heinrich, G. Luisoni, P. Mastrolia, E. Mirabella, G. Ossola et al., Eur. Phys. J. C 74(8), 3001 (2014). arXiv: 1404.7096

54. R.V. Harlander, S. Liebler, T. Zirke, JHEP 1402, 023 (2014). arXiv: 1307.8122

55. O. Brein, R.V. Harlander, T.J.E. Zirke, Comput. Phys. Commun. 184, 998 (2013). arXiv:1210.5347

56. D. Eriksson, J. Rathsman, O. Stål, Comput. Phys. Commun. 181, 189 (2010). arXiv:0902.0851

57. V. Khachatryan et al. (CMS Collaboration), JHEP 1510, 144 (2015). arXiv: 1504.00936

58. G. Aad et al. (ATLAS Collaboration), Eur. Phys. J. C 76(1), 45 (2016). doi:10.1140/epjc/s10052-015-3820-z

59. R.V. Harlander, S. Liebler, H. Mantler, Comput. Phys. Commun. 184, 1605 (2013). arXiv:1212.3249

60. A. Bredenstein, A. Denner, S. Dittmaier, M.M. Weber, JHEP 0702, 080 (2007). arXiv:hep-ph/0611234

61. A. Bredenstein, A. Denner, S. Dittmaier, M.M. Weber, Phys. Rev. D 74, 013004 (2006). arXiv:hep-ph/0604011

62. T. Hahn, Comput. Phys. Commun. 140, 418 (2001). arXiv:hep-ph/0012260

63. T. Hahn, M. Perez-Victoria, Comput. Phys. Commun. 118, 153 (1999). arXiv:hep-ph/9807565

64. P. Nogueira, J. Comput. Phys. 105, 279 (1993)

65. J.A.M. Vermaseren, arXiv:math-ph/0010025

66. J. Kuipers, T. Ueda, J.A.M. Vermaseren, J. Vollinga, Comput. Phys. Commun. 184, 1453 (2013). arXiv:1203.6543

67. G. Cullen, M. Koch-Janusz, T. Reiter, Comput. Phys. Commun. 182, 2368 (2011). arXiv: 1008.0803

68. T. Reiter, Comput. Phys. Commun. 181, 1301 (2010). arXiv:0907.3714

69. P. Mastrolia, E. Mirabella, T. Peraro, JHEP 1206, 095 (2012). arXiv:1203.0291 [JHEP 1211, 128 (2012)]

70. H. van Deurzen, G. Luisoni, P. Mastrolia, E. Mirabella, G. Ossola, T. Peraro, JHEP 1403, 115 (2014). arXiv:1312.6678 
71. T. Peraro, Comput. Phys. Commun. 185, 2771 (2014). arXiv: 1403.1229

72. G. Ossola, C.G. Papadopoulos, R. Pittau, Nucl. Phys. B 763, 147 (2007). arXiv:hep-ph/0609007

73. P. Mastrolia, G. Ossola, C.G. Papadopoulos, R. Pittau, JHEP 0806, 030 (2008). arXiv:0803.3964

74. G. Ossola, C.G. Papadopoulos, R. Pittau, JHEP 0805, 004 (2008). arXiv:0802.1876

75. P. Mastrolia, G. Ossola, T. Reiter, F. Tramontano, JHEP 1008, 080 (2010). arXiv:1006.0710

76. G. Heinrich, G. Ossola, T. Reiter, F. Tramontano, JHEP 1010, 105 (2010). arXiv:1008.2441

77. T. Binoth, J.-P. Guillet, G. Heinrich, E. Pilon, T. Reiter, Comput. Phys. Commun. 180, 2317 (2009). arXiv:0810.0992

78. G. Cullen, J.P. Guillet, G. Heinrich, T. Kleinschmidt, E. Pilon, T. Reiter, M. Rodgers, Comput. Phys. Commun. 182, 2276 (2011). arXiv: 1101.5595
79. J.P. Guillet, G. Heinrich, J.F. von Soden-Fraunhofen, Comput. Phys. Commun. 185, 1828 (2014). arXiv:1312.3887

80. A. van Hameren, Comput. Phys. Commun. 182, 2427 (2011). arXiv: 1007.4716

81. F. Maltoni, T. Stelzer, JHEP 0302, 027 (2003). arXiv:hep-ph/0208156

82. J. Alwall et al., JHEP 0709, 028 (2007). arXiv:0706.2334

83. H.E. Haber, O. Stål, Eur. Phys. J. C 75(10), 491 (2015). arXiv: 1507.04281

84. P.M. Ferreira, J.F. Gunion, H.E. Haber, R. Santos, Phys. Rev. D 89(11), 115003. arXiv: 1403.4736

85. P.M. Ferreira, R. Guedes, J.F. Gunion, H.E. Haber, M.O.P. Sampaio, R. Santos, arXiv:1410.1926

86. J. Gao et al., Phys. Rev. D 89(3), 033009 (2014). arXiv:1302.6246

87. G. Aad et al. (ATLAS Collaboration), JHEP 1601, 032 (2016). doi:10.1007/JHEP01(2016)032 